\title{
ON FREE NETS OVER MINKOWSKI SPACE
}

\author{
Hellmut BaumGäRTEL* \\ Mathematisches Institut der Universität Potsdam, Am Neuen Palais 10, Postfach 601 553, \\ 14415 Potsdam, Germany \\ MATTHIAS JURKE ${ }^{\dagger}$ \\ Technische Universität Berlin, Fachbereich Mathematik, MA 8-5, Straße des 17. Juni 136, \\ 10623 Berlin, Germany (e-mail: jurke@sfb288.math.tu-berlin.de) \\ and \\ FERNANDO LLEDÓ $\ddagger$ \\ Technische Universität Berlin, Institut für Theoretische Physik, PN 7-1, Hardenbergstraße 36 , \\ 10623 Berlin, Germany (e-mail: flm0433@w42lzrz.physik.tu-berlin.de)
}

\begin{abstract}
Using standard results on CAR- and CCR-theory and on representation theory of the Poincare group a direct way to construct nets of local $\mathrm{C}^{*}$-algebras satisfying Haag-Kastler's axioms is given. No explicite use of any field operator or of any concrete representation of the algebra is made. The nets are associated to models of mass $m \geq 0$ and arbitrary spin or helicity. Finally, Fock states satisfying the spectrality condition are specified.
\end{abstract}

\section{Introduction}

The aim of the present paper is to give an abstract construction of a causal and covariant net of local $C^{*}$-algebras $[1,2]$ associated to free massive and massless models with arbitrary spin and helicity, respectively. The net will be constructed directly, i.e. no field operator or any concrete representation of the algebra will be used explicitly. (Compare with the approach in [3].) The quantization procedure does not use any underlying classical theory either. For this reasons we will call the result of this construction a free net. Its relation to the standard net of von Neumann algebras obtained from the free fields is still open.

*Partly supported by DFG; SFB 288: "Differentialgeometrie und Quantenphysik".

†Supported by DFG; SFB 288: "Differentialgeometrie und Quantenphysik".

${ }^{\ddagger}$ Supported by grant of the Spanish Ministry of Education/CICYT. Present address: Mathematisches Institut der Universität Potsdam, Am Neuen Palais 10, Postfach 601 553, 14415 Potsdam, Germany. 
The construction we propose is based on the well-known facts from the CARand CCR-theory $[4,5,6,7,8]$ and on standard results of the classification of the unitary irreducible representation (UIR) of the Poincare group $[9,10]$. Indeed, one can interpret certain combinations of the Hilbert spaces associated to the parameters $\{m \geq 0, s, \pm\}$ as the reference spaces of CAR- and CCR-algebras. Then, the local algebras that satisfy Haag-Kastler's axioms are specified as $\mathrm{C}^{*}$-subalgebras of the CAR- and CCR-algebras constructed before. In this construction all spins (or helicities) are treated at the same formal level, so that no concrete spin or helicity degree is emphasized. Within the same strategy alternative construction procedures for the net of local algebras were defined and compared in [11], [12] and [13].

The paper is organized in 9 sections: In Section 2 the construction of local algebras associated to fermionic and bosonic models, that satisfy Haag-Kastler's axioms, is reduced to the construction of certain nets of linear submanifolds of the respective reference spaces.

Part A corresponds to the study of massive models. In Section A.1 the first definitions that characterize the concrete models are given and some technical lemmas are proved. In Section A. 2 the CAR- and CCR-algebras for the models are constructed, and in Section A.3 the two main theorems of existence of the nets of linear submanifolds for the Fermi and Bose models are stated and proved. In the next section Fock states for these models are defined for which the spectrality condition is satisfied.

Part B analyses the massless models: In Section B.1 we describe some pecularities of the representation theory of massless models. We also present some results concerning the theory of induced representations. In Section B.2 the factor spaces $\mathfrak{H}_{ \pm}^{\prime}$ are constructed and some of its properties are proved. These spaces are associated to massless models with complementary helicities. In the next section the causal and covariant nets associated to fermionic and bosonic models are constructed. In the concluding section Fock states satisfying the spectrality condition are defined for these cases.

\section{Nets of local linear submanifolds of the reference spaces}

It is a well-known result that once a complex Hilbert space $h_{\mathrm{F}}$ and an anti-unitary involution $\Gamma$ on $h_{\mathrm{F}}$ are fixed, then an algebraically unique $\mathrm{C}^{*}$-algebra can be constructed. It is denoted by $\operatorname{CAR}\left(h_{\mathrm{F}}, \Gamma\right)$ [4]. Analogously, $\operatorname{CCR}\left(h_{\mathrm{B}}, \sigma\right)$ denotes the algebraically unique $C^{*}$-algebra over a real-linear space $h_{\mathrm{B}}$, where a non-degenerated symplectic form $\sigma$ is defined [8]. It is therefore natural to construct a net of local algebras using a certain net of linear submanifolds of the respective spaces. The precise properties that the net of linear submanifolds must satisfy in order to generate a correct net of local algebras are stated in this section.

We denote by $\mathcal{O}, \mathcal{O}_{1}, \mathcal{O}_{2} \subset \mathbb{R}^{4}$ open and bounded subsets of the Minkowski space and by $\widetilde{\mathfrak{P}_{+}^{\dagger}}$ the universal covering group of the proper orthochronous component of the Poincaré group. The construction of the local algebras will be based on the following facts (see e.g. $[7$, p. $140 \mathrm{ff}$.$] ):$ 
- Let $\Gamma$ be an anti-unitary involution on a complex Hilbert space $h_{\mathrm{F}}$ and let

$$
\mathcal{O} \longmapsto M_{\mathrm{F}}(\mathcal{O})
$$

be a net of complex-linear submanifolds of $h_{\mathrm{F}} . V_{\mathrm{F}}$ is a strongly continuous unitary representation of $\widetilde{\mathfrak{P}_{+}^{\dagger}}$ on $h_{\mathrm{F}}$ with $\Gamma V_{\mathrm{F}}(g)=V_{\mathrm{F}}(g) \Gamma$. Assume that for any $\mathcal{O}, \mathcal{O}_{1}$ and $\mathcal{O}_{2}$ the following properties are satisfied:

$(0)_{\mathrm{F}} M_{\mathrm{F}}(\mathcal{O})$ is $\Gamma$-invariant (i.e. $\Gamma M_{\mathrm{F}}(\mathcal{O}) \subset M_{\mathrm{F}}(\mathcal{O})$ ),

$(\mathrm{I})_{\mathrm{F}} \mathcal{O}_{1} \subseteq \mathcal{O}_{2}$ implies $M_{\mathrm{F}}\left(\mathcal{O}_{1}\right) \subseteq M_{\mathrm{F}}\left(\mathcal{O}_{2}\right)$,

(II) $M_{\mathrm{F}}(g \mathcal{O})=V_{\mathrm{F}}(g) M_{\mathrm{F}}(\mathcal{O}), g \in \widehat{\mathfrak{P}_{+}^{\dagger}}$,

(III) $)_{\mathrm{F}} \mathcal{O}_{1} \perp \mathcal{O}_{2}$ implies $M_{\mathrm{F}}\left(\mathcal{O}_{1}\right) \perp M_{\mathrm{F}}\left(\mathcal{O}_{2}\right)$,

where $\mathcal{O}_{1} \perp \mathcal{O}_{2}$ means that $\mathcal{O}_{1}$ and $\mathcal{O}_{2}$ are spacelike separated, and $M_{\mathrm{F}}\left(\mathcal{O}_{1}\right) \perp M_{\mathrm{F}}\left(\mathcal{O}_{2}\right)$ means that $M_{\mathrm{F}}\left(\mathcal{O}_{1}\right)$ and $M_{\mathrm{F}}\left(\mathcal{O}_{2}\right)$ are orthogonal in $h_{\mathrm{F}}$. Let $\mathbb{Z}_{2}=\{\iota, \theta\}$, where $\iota$ is the identical automorphism and $\theta:=\alpha_{\vartheta}$ is the Bogoliubov automorphism of $\operatorname{CAR}\left(h_{\mathrm{F}}, \Gamma\right)$ which corresponds to $\vartheta_{\varphi}:=-\varphi$. As usual $A(\varphi), \varphi \in h_{\mathrm{F}}$, denote the "canonical generators" of $\operatorname{CAR}\left(h_{\mathrm{F}}, \Gamma\right) . \mathcal{B}^{\mathbb{Z}_{2}}$ means a fixed point subalgebra of $\mathcal{B}$ w.r.t. the automorphism group $\mathbb{Z}_{2}$. Then the algebras

$$
\mathcal{A}_{\mathrm{F}}(\mathcal{O}):=\mathrm{C}^{*}\left(\left\{A(\varphi): \varphi \in M_{\mathrm{F}}(\mathcal{O})\right\}\right)^{\mathbb{Z}_{2}}
$$

form an isotone, covariant and causal net of $\mathrm{C}^{*}$-algebras, i.e. for any $\mathcal{O}, \mathcal{O}_{1}$ and $\mathcal{O}_{2}$ one has:

(i) $\mathcal{O}_{1} \subseteq \mathcal{O}_{2}$ implies $\mathcal{A}\left(\mathcal{O}_{1}\right) \subseteq \mathcal{A}\left(\mathcal{O}_{2}\right)$,

(ii) $\mathcal{A}(g \mathcal{O})=\alpha_{g} \mathcal{A}(\mathcal{O}), g \in \widehat{\mathfrak{P}_{+}^{\dagger}}$,

(iii) $)_{\mathrm{F}} \mathcal{O}_{1} \perp \mathcal{O}_{2}$ implies that $\mathcal{A}\left(\mathcal{O}_{1}\right)$ and $\mathcal{A}\left(\mathcal{O}_{2}\right)$ commute elementwise,

where $\alpha_{g}$ denotes the Bogoliubov automorphism that corresponds to $V_{F}(g)$.

- Let $\sigma(\cdot, \cdot)$ be a non-degenerated symplectic form on a real-linear space $h_{\mathrm{B}}$. Let

$$
\mathcal{O} \longmapsto M_{\mathrm{B}}(\mathcal{O})
$$

be a net of real-linear submanifolds of $h_{\mathrm{B}}$ and let $V_{\mathrm{B}}$ be a symplectic representation of $\widetilde{P_{+}^{\top}}$. Assume that for any $\mathcal{O}, \mathcal{O}_{1}$ and $\mathcal{O}_{2}$ the following conditions are satisfied:

$(\mathrm{I})_{\mathrm{B}} \mathcal{O}_{1} \subseteq \mathcal{O}_{2}$ implies $M_{\mathrm{B}}\left(\mathcal{O}_{1}\right) \subseteq M_{\mathrm{B}}\left(\mathcal{O}_{2}\right)$,

(II) $M_{\mathrm{B}}(g \mathcal{O})=V_{\mathrm{B}}(g) M_{\mathrm{B}}(\mathcal{O}), g \in \overline{\mathfrak{P}_{+}^{\dagger}}$,

(III) $)_{\mathrm{B}} \mathcal{O}_{1} \perp \mathcal{O}_{2}$ implies $M_{\mathrm{B}}\left(\mathcal{O}_{1}\right) \perp M_{\mathrm{B}}\left(\mathcal{O}_{2}\right)$,

where $M_{\mathrm{B}}\left(\mathcal{O}_{1}\right) \perp M_{\mathrm{B}}\left(\mathcal{O}_{2}\right)$ means that $M_{\mathrm{B}}\left(\mathcal{O}_{1}\right)$ and $M_{\mathrm{B}}\left(\mathcal{O}_{2}\right)$ are symplectic orthogonal. As usual $W(\varphi), \varphi \in h_{\mathrm{B}}$, denote the "Weyl elements" of $\operatorname{CCR}\left(h_{\mathrm{B}}, \sigma\right)$. In this case the algebras

$$
\mathcal{A}_{\mathrm{B}}(\mathcal{O}):=\mathrm{C}^{*}\left(\left\{W(\varphi): \varphi \in M_{\mathrm{B}}(\mathcal{O})\right\}\right)
$$


form an isotone, covariant and causal net of $\mathrm{C}^{*}$-algebras, i.e. for any $\mathcal{O}, \mathcal{O}_{1}$ and $\mathcal{O}_{2}$ one has:

(i) $\mathcal{B}_{\mathrm{B}} \subseteq \mathcal{O}_{2}$ implies $\mathcal{A}\left(\mathcal{O}_{1}\right) \subseteq \mathcal{A}\left(\mathcal{O}_{2}\right)$,

(ii) $\mathcal{B} \mathcal{A}(g \mathcal{O})=\alpha_{g} \mathcal{A}(\mathcal{O}), g \in \mathfrak{P}_{+}^{\uparrow}$,

(iii) $\mathcal{O}_{1} \perp \mathcal{O}_{2}$ implies that $\mathcal{A}\left(\mathcal{O}_{1}\right)$ and $\mathcal{A}\left(\mathcal{O}_{2}\right)$ commute elementwise,

where $\alpha_{g}$ denotes the Bogoliubov automorphism that corresponds to $V_{\mathrm{B}}(g)$.

REMARK: By these propositions the problem of constructing covariant and causal nets of local $\mathrm{C}^{*}$-algebras (within the framework of CAR-and CCR-theory) is shifted to the construction of corresponding nets of linear submanifolds of the reference spaces.

Part A: The massive case $(m>0)$

\section{A.1. Representations, embeddings and their mutual relations}

We consider the following representations of $\widehat{\mathfrak{P}_{+}^{\dagger}}$ on $\mathrm{C}_{0}^{\infty}\left(\mathbb{R}^{4}, \mathcal{H}\right)$ and on $h_{m}:=$ $\mathrm{L}^{2}\left(H_{m}^{+}, \mathcal{H}, \mu(\mathrm{d} p)\right)$ :

$$
\begin{array}{lll}
\left(T_{1}(g) f\right)(x):=D_{A} f\left(A_{A}^{-1}(x-a)\right) & \text { for } & f \in C_{0}^{\infty}\left(\mathbb{R}^{4}, \mathcal{H}\right), \\
\left(V_{1}(g) \varphi\right)(p):=e^{-i p a} D_{A \varphi}\left(A_{A}^{-1} p\right) & \text { for } & \varphi \in h_{m}, \\
\left(V_{2}(g) \varphi\right)(p):=e^{i p a} D_{A \varphi}\left(A_{A}^{-1} p\right) & \text { for } & \varphi \in h_{m},
\end{array}
$$

where $g=(A, a) \in \widehat{\mathfrak{P}_{+}^{\top}}=\operatorname{SL}(2, \mathbb{C})(\widehat{S}) \mathbb{R}^{4}, A \mapsto D_{A}:=\otimes A$ is a finite-dimensional representation of $\operatorname{SL}(2, \mathbb{C})$ over $\mathcal{H}:=\operatorname{Sym}\left(\otimes \mathbb{C}^{2}\right)$ of ${ }^{1}$ the type $(n, 0)$ and $A_{A}$ is the corresponding Lorentz transformation for the matrices $\pm A \in \operatorname{SL}(2, \mathbb{C})$. With respect to the scalar product

$$
\langle\varphi, \psi\rangle_{\beta_{+}}:=\int_{I_{m}^{+}}\left(\varphi(p), \beta_{+}(p) \psi(p)\right)_{\mathcal{H}} \mu(\mathrm{d} p) \quad \text { for all } \quad \varphi, \psi \in h_{m}
$$

the representations $V_{1}$ and $V_{2}$ are unitary and irreducible, where

$$
\beta_{+}(p):=D_{P^{+}}=\bigotimes^{n} P^{+}, \quad P^{+}=\frac{1}{m}\left(p_{0} \sigma_{0}-\sum_{i=1}^{3} p_{i} \sigma_{i}\right)
$$

and $\sigma_{\mu}$ are the Pauli matrices, i.e.

\footnotetext{
${ }^{1}$ Sym denotes the symmetrization operator.
} 


$$
\sigma_{0}=\left(\begin{array}{ll}
1 & 0 \\
0 & 1
\end{array}\right) . \quad \sigma_{1}=\left(\begin{array}{ll}
0 & 1 \\
1 & 0
\end{array}\right), \quad \sigma_{2}=\left(\begin{array}{rr}
0 & -i \\
i & 0
\end{array}\right), \quad \sigma_{3}=\left(\begin{array}{rr}
1 & 0 \\
0 & -1
\end{array}\right) .
$$

LEMMA A.1.1. $V_{1}$ and $V_{2}$ are anti-unitarily equivalent, i.e. there exists an anti-linear mapping (unique up to a phase) $\Gamma_{1}: h_{m} \rightarrow h_{m}$ that satisfies $\left\langle\Gamma_{1} \varphi, \Gamma_{1} \psi\right\rangle_{\beta_{+}}=\left\langle u^{\prime}, \varphi\right\rangle_{i_{+}}$ for all $;, \psi \in h_{m}$ and $\Gamma_{1} V_{1}=V_{2} \Gamma_{1}$.

Proof: Let $\Gamma_{0}$ be an anti-unitary involution on $\mathcal{H}$. Define $E:=\left(\begin{array}{rr}0 & 1 \\ -1 & 0\end{array}\right) \in \mathrm{SU}(2)$ and $H_{\mu}:=(m+P) / \sqrt{2 m\left(m+p_{0}\right)}$, where $P=\sum_{\mu} p_{\mu} \sigma_{\mu}$. By direct computation it can be shown that $\Gamma_{1}:=D_{H_{p}} D_{E}^{-1} \Gamma_{0} D_{H_{p}^{-1}}$ satisfies all the mentioned properties.

The uniqueness (up to a phase) of $\Gamma_{1}$ is a consequence of the irreducibility of $V_{1}$ and $V_{2}$.

REMARK A.1.2: The existence of $\Gamma_{1}$ is based on the fact that the self-representation and the conjugate representation of SU (2) are equivalent. $E$ is precisely the unitarity intertwining between both representations. For massless models the corresponding little group will not have this property, so that the following construction of Fermi models in Section A.2 can not be extended directly in order to include the massless case. (Compare with the definition of $\Gamma$ in Subsection B.3.2.1.)

The proof of the covariance property of the net (see (II) $)_{\mathbb{B}, F}$ in the preceding section) will be based on the following

REMARK A.1.3: The linear embeddings $\mathfrak{J}_{ \pm}: \mathrm{C}_{0}^{\infty}\left(\mathbb{R}^{4}, \mathcal{H}\right) \rightarrow h_{m}$, defined by $\left(\Im_{ \pm} f\right)(p):=\hat{f}( \pm p),{ }^{2}$ satisfy the equations

$$
\mathfrak{I}_{+} T_{1}(g)=V_{1}(g) \mathfrak{I}_{+}, \quad \mathfrak{I}_{-} T_{1}(g)=V_{2}(g) \mathfrak{J} \quad \text { for all } \quad g \in \widehat{\mathfrak{P}_{+}^{\top}} .
$$

The causality (see (III) $)_{\mathrm{B}, \mathrm{F}}$ in the preceding section) of the net will be based on the following result:

LEMMA A.1.4. Let $x \in \mathbb{R}^{4}$ be a spacelike vector (i.e. $x^{2}=x_{0}^{2} \quad x_{1}^{2}-x_{2}^{2} \quad x_{3}^{2}<0$ ). With the notation of the beginning of this section one has

$$
\begin{aligned}
& \int_{H_{m}^{+}}\left(e^{i p x}-e^{-i p x}\right) \beta_{+}(p) \mu(\mathrm{d} p)=0, \quad n \text { even, } \\
& \int_{I I_{m}^{+}}\left(e^{i p x}+e^{-i p x}\right) \beta_{+}(p) \mu(\mathrm{d} p)=0, \quad n \text { odd } .
\end{aligned}
$$

\footnotetext{
${ }^{2}$ The hat ${ }^{-}$means the Fourier transformation restricted to the positive mass shell $H_{m}^{+}$.
} 
Proof: It is well-known that for $x^{2}<0$ the two-point function

$$
\Delta(x)=\int_{H_{m}^{+}} e^{-i p x} \mu(\mathrm{d} p)
$$

is an even $\mathrm{C}^{\infty}$ function, i.e. $\Delta(x)=\Delta(-x)$ (see [15, pp. 71 and 107]). Let $\alpha$ be a multi-index and $|\alpha|:=\alpha_{0}+\alpha_{1}+\alpha_{2}+\alpha_{3}$. Then

$$
\begin{array}{r}
\left.\frac{\partial^{|\alpha|} \Delta}{\partial x^{\alpha}}\right|_{x}=(-i)^{\alpha_{0}-\alpha_{1}-\alpha_{2}-\alpha_{3}} \int_{H_{m}^{+}} p^{\alpha} e^{-i p x} \mu(\mathrm{d} p) \\
\text { is an }\left\{\begin{array}{l}
\text { even function, if }|\alpha| \text { is even } \\
\text { odd function, if }|\alpha| \text { is odd. }
\end{array}\right.
\end{array}
$$

But from Eqs. (9) it follows that the matrix elements of $\beta_{+}(p)$ are homogeneous polynomials of degree $n$ in $p_{\mu} \cdot{ }^{3}$ Equations (12) and (13) are therefore true.

Although in the massless case $(m=0) \beta_{+}(p)$ has a different form than in Eq. (9) (see Section B.2.1), analogous equations as in (12) and (13) can be proved for $m=0$ using the same arguments as above, since the corresponding matrix elements are, again, homogeneous polynomials of degree $n$ in $p_{\mu}$.

\section{A.2. CAR- and CCR-algebras}

\section{A.2.1. The Fermi case}

As the complex Hilbert space we consider $h_{\mathrm{F}}:=h_{m} \oplus h_{m}$ with the scalar product defined by $\langle\varphi, \psi\rangle_{\mathrm{F}}:=\left\langle\varphi_{1}, \psi_{1}\right\rangle_{\beta_{+}}+\left\langle\varphi_{2}, \psi_{2}\right\rangle_{\beta_{+}}$, for all $\varphi=\varphi_{1} \oplus \varphi_{2}, \psi=\psi_{1} \oplus \psi_{2} \in h_{\mathrm{F}}$. Using the expression for $\Gamma_{1}$ given in the proof of Lemma A.1.1, we define on $h_{\mathrm{F}}$ the following involution: $\Gamma\left(\varphi_{1} \oplus \varphi_{2}\right):=\Gamma_{1}^{-1} \varphi_{2} \oplus \Gamma_{1} \varphi_{1}$, which satisfies all the required properties (i.e. $\Gamma$ is anti-linear, $\Gamma^{2}=1$ and $\langle\Gamma \varphi, \Gamma \psi\rangle_{\mathrm{F}}=\langle\psi, \varphi\rangle_{\mathrm{F}}$, for all $\varphi, \psi \in h_{\mathrm{F}}$ ). $\mathrm{CAR}\left(h_{\mathrm{F}}, \Gamma\right)$ is therefore uniquely given.

\section{A.2.2. The Bose case}

We interpret $h_{\mathrm{B}}:=h_{n} \oplus h_{m}$ as a real-linear space with a non-degenerate form defined by

$$
\langle\varphi, \psi\rangle_{\mathrm{B}}:=\left\langle\varphi_{1}, \psi_{1}\right\rangle_{\beta_{+}}-\left\langle\varphi_{2}, \psi_{2}\right\rangle_{\beta_{+}} .
$$

The symplectic form is $\sigma(\cdot, \cdot):=\operatorname{Im}(\cdot, \cdot\rangle_{\mathrm{B}} \cdot \operatorname{CCR}\left(h_{B}, \sigma\right)$ is then uniquely determined.

\footnotetext{
${ }^{3}(n, 0)$ characterizes the representation $D$ (see the beginning of this section).
} 


\section{A.3. Existence theorems for local algebras}

We define an embedding $\mathfrak{I}: \mathrm{C}_{0}^{\infty}\left(\mathbb{R}^{4}, \mathcal{H}\right) \rightarrow h_{\mathrm{F}, \mathrm{B}}$ by $\mathfrak{I}:=\mathfrak{I}_{+} \oplus \mathfrak{I}_{-}$(see $\operatorname{Re}$ mark A.1.3) and the representations of $\widetilde{\mathfrak{P}_{+}^{\dagger}}$ on $h_{\mathrm{F}, \mathrm{B}}$ by $V_{\mathrm{F}, \mathrm{B}}(g):=V_{1}(g) \oplus V_{2}(g)$.

\section{A.3.1. The Fermi case}

Define a net of linear submanifolds of $h_{\mathrm{F}}$ by

$$
\mathbb{R}^{4} \supset \mathcal{O} \vdash \rightarrow M_{\mathrm{F}}(\mathcal{O}):=\operatorname{span}\left\{\mathfrak{I} f: \quad f \in \mathbb{C}_{0}^{\infty}\left(\mathbb{R}^{4}, \mathcal{H}\right), \operatorname{supp} f \subset \mathcal{O}\right\},
$$

for each open and bounded region $\mathcal{O} \subset \mathbb{R}^{4}$.

LEMMA A.3.1. The equations $\Gamma V_{\mathrm{F}}(g)=V_{\mathrm{F}}(g) \Gamma$ and $\mathfrak{I} T_{1}(g)=V_{\mathrm{F}}(g) \mathfrak{I}$ are valid for all $g \in \widehat{\mathfrak{P}_{+}^{\dagger}}$.

Proof: These statements are direct consequences of the definitions of $\Gamma, \Gamma_{1}, V_{\mathrm{F}}$ and $T_{1}$.

THEOREM A.3.2. The net $\mathcal{O} \mapsto M_{\mathrm{F}}(\mathcal{O})$ satisfies the properties $(0)_{\mathrm{F}},(\mathrm{I})_{\mathrm{F}},(\mathrm{II})_{\mathrm{F}}$ and (III) $\mathrm{F}$ of Section 2.

Proof: To prove $(0)_{\mathrm{F}}$, we start with the case $n=1$. With this assumption and using the definitions of $H_{p}$ and $F$ (see proof of Lemma A.1.1) we get

$$
\Gamma_{1}=\frac{1}{m} \Gamma_{0}(E P)^{t}=\frac{1}{m} \Gamma_{0}\left(\begin{array}{cc}
p_{1}+i p_{2} & -\left(p_{0}+p_{3}\right) \\
p_{0}-p_{3} & -p_{1}+i p_{2}
\end{array}\right),
$$

and $I_{1}^{-1}=-\Gamma_{1}$. (Here, the exponent $t$ denotes the transposition of the matrix.) A straightforward calculation shows that for all $\mathfrak{I} f \in M_{\mathrm{F}}(\mathcal{O})$, with $f=\left(\begin{array}{l}f_{1} \\ f_{2}\end{array}\right)$, $\Gamma \mathfrak{I} f=\mathfrak{J} h$, where

$$
h=\frac{i}{m}\left(\begin{array}{c}
\left(\partial_{1}-i \partial_{2}\right) \bar{f}_{1}-\left(\partial_{0}+\partial_{3}\right) \bar{f}_{2} \\
\left(\partial_{0}-\partial_{3}\right) \bar{f}_{1}-\left(\partial_{1}+i \partial_{2}\right) \bar{f}_{2}
\end{array}\right)
$$

and therefore $\mathfrak{I} h \in M_{\mathrm{F}}(\mathcal{O})$. This fact, together with the standard properties of the symmetrization operator on $\stackrel{n}{\otimes}^{2}$ and of the embeddings $\mathfrak{J}_{+}, \mathfrak{J}$, assure the $\Gamma$-invariance of $M_{\mathrm{F}}(\mathcal{O})$ for arbitrary $n$.

The isotony property $(\mathrm{I})_{\mathrm{F}}$ is trivial from the definition of the net. To check the covariance, define for any $I f \in M_{\mathrm{F}}(\mathcal{O})$ an element $k:=T_{1}(g) f \in \mathrm{C}_{0}^{\infty}\left(\mathbb{R}^{4}, \mathcal{H}\right)$ such that supp $k \subset g \mathcal{O}$. We have $V_{\mathrm{F}}(g) \mathfrak{I} f=\mathfrak{I} T_{1}(g) f=\mathfrak{I} k$. To prove the other inclusion, define for any $\Im k \in M_{\mathrm{F}}(g \mathcal{O})$ an element $f:=T_{1}\left(g^{-1}\right) k \in \mathrm{C}_{0}^{\infty}\left(\mathbb{R}^{4}, \mathcal{H}\right)$ such that $\operatorname{supp} f \subset \mathcal{O}$. In this case it can easily be verified that $\mathfrak{I} k=V_{\mathrm{F}}(g) \mathfrak{I} f$. Finally, Lemma A.3.1 assures the covariance of the net. 
To prove the causality property, choose $I f$ and $\mathfrak{I} h$ such that $\operatorname{supp} f \perp \operatorname{supp} h$. We have

$$
\left\langle\mathfrak{I}_{-} f, \mathfrak{I}_{-} h\right\rangle_{\beta_{1}}=\int_{H_{m}^{+}}\left(\widehat{f}(-p), \beta_{+}(p) \widehat{h}(-p)\right)_{\mathcal{H}} \mu(\mathrm{d} p) .
$$

Therefore

$$
\begin{aligned}
\langle\Im f, I h\rangle_{\mathrm{F}}= & \int_{H_{m}^{+}}\left(\int_{\mathbb{R}^{4}} e^{-i p x} f(x) \mathrm{d}^{4} x, \beta_{+}(p) \int_{\mathbb{P}^{4}} e^{-i p y} h(y) \mathrm{d}^{4} y\right)_{\mathcal{H}} \mu(\mathrm{d} p)+ \\
& +\int_{H_{m}^{+}}\left(\int_{y^{4}} e^{i p x x} f(x) \mathrm{d}^{4} x, \beta_{+}(p) \int_{\mathbb{E}^{4}} e^{i p y} h(y) \mathrm{d}^{4} y\right)_{\mathcal{H}} \mu(\mathrm{d} p) \\
= & \int_{\mathbb{R}^{4}} \int_{\mathbb{R}^{4}}\left(f(x),\left[\int_{I I_{m}^{+}}\left(e^{i p(x-y)}+e^{-i p(p-y)}\right) \beta_{+}(p) \mu(\mathrm{d} p)\right] h(y)\right)_{\mathcal{H}} \mathrm{d}^{4} x \mathrm{~d}^{4} y=0,
\end{aligned}
$$

from Eq. (13).

Following Section 2 we have

THEOREM A.3.3. The net of $\mathrm{C}^{*}$-algebras defined by

$$
\mathbb{R}^{4} \supset \mathcal{O} \longmapsto \mathcal{A}_{\mathrm{F}}(\mathcal{O}):=\mathrm{C}^{*}\left(\left\{A(f): \quad f \in M_{\mathrm{F}}(\mathcal{O})\right\}\right)^{\sigma_{2}}
$$

satisfies the properties $(\mathrm{i})_{\mathrm{F}},(\mathrm{ii})_{\mathrm{F}}$ and $(\mathrm{iii})_{\mathrm{F}}$.

\section{A.3.2. The Bose case}

In an analogous way we define the net of linear submanifolds of $k_{B}$ by

$$
\mathbb{R}^{4} \supset \mathcal{O} \longmapsto M_{\mathrm{B}}(\mathcal{O}):=\operatorname{span}\left\{\mathfrak{X} f: f \in \mathrm{C}_{0}^{\infty}\left(\mathbb{R}^{4}, \mathcal{H}\right), \operatorname{supp} f \subset \mathcal{O}\right\},
$$

for each open and bounded region $\mathcal{O} \subset \mathbb{R}^{4}$.

LemmA A.3.4. The equations $\sigma\left(V_{\mathrm{B}}(g)_{\varphi}, V_{\mathrm{B}}(g) \psi\right)=\sigma(\varphi, \psi)$ and $\mathfrak{I} T_{1}(g)=V_{\mathrm{B}}(g) \mathfrak{I}$ are valid for any $g \in \widehat{\mathfrak{P}_{+}^{\dagger}}$ and $\varphi, \psi \in h_{\mathrm{B}}$.

Proof: These statements are direct consequences of the definitions of $\sigma, V_{B}$ and $T_{1}$.

THEOREM A.3.5. The net $\mathcal{O} \mapsto M_{\mathrm{B}}(\mathcal{O})$ satisfies the properties (I) $)_{\mathrm{B}}$, (II) $)_{\mathrm{B}}$ and (III) of Section 2.

Proof: The isotony property is again trivial. The covariance $(\mathrm{II})_{\mathrm{B}}$ can be verified using Lemma A.3.4 and analogous arguments as in Theorem A.3.2. 
For the causality (III) $)_{B}$ choose $I f$ and $I h$ such that supp $f \perp$ supp $h$. We have

$$
\begin{aligned}
& \langle\Im \mathcal{J}, \Im h\rangle_{\mathrm{B}}=\int_{H_{w}^{+}}\left(\int_{\mathbb{R}^{4}} e^{-i p x} f(x) \mathrm{d}^{4} x, \beta_{+}(p) \int_{\mathbb{F}^{4}} e^{-i p y} h(y) \mathrm{d}^{4} y\right)_{\mathcal{H}} \mu(\mathrm{d} p)- \\
& -\int_{H_{m}^{+}}\left(\int_{\mathbb{F}^{4}} e^{i p x} f(x) \mathrm{d}^{4} x, \beta_{+}(p) \int_{\vartheta^{4}} e^{i p y} h(y) \mathrm{d}^{4} y\right)_{\mathcal{H}^{4}} \mu(\mathrm{d} p) \\
& =\int_{\mathrm{X}^{-4}} \int_{\mathrm{z}^{-1}}\left(\int(x),\left[\int_{H_{m}^{+}}\left(e^{i p(x-y)}-e^{-i p(x-y)}\right) \beta_{+}(p) \mu(\mathrm{d} p)\right] h(y)\right) \mathrm{d}^{4} x \mathrm{~d}^{4} y=0 .
\end{aligned}
$$

using Eq. (12). ${ }^{4}$

Again following Section 2 we have

THEOREM A.3.6. The net of $\mathrm{C}^{*}$-algebras defined by

$$
\mathbb{R}^{4} \supset \mathcal{O} \longmapsto \mathcal{A}_{\mathrm{B}}(\mathcal{O}):=\mathrm{C}^{*}\left(\left\{W(f): f \in M_{\mathrm{B}}(\mathcal{O})\right\}\right)
$$

satisfies the properties $(\mathrm{i})_{\mathrm{B}}$, $(\mathrm{ii})_{\mathrm{B}}$ and $(\mathrm{iii})_{\mathrm{B}}$.

\section{A.4. Fock states and spectrality condition}

Since the representations $V_{1}$ and $V_{2}$ (from which the transformations $V_{\mathrm{F} . \mathrm{B}}$ that generate Bogoliubov automorphisms can be constructed) are anti-unitarily equivalent, ${ }^{5}$ it is natural to ask if there exists a Fock state for which the spectrality condition is satisfied. To answer affirmatively this question, it will be useful in the Fermi case to characterize the Fock states via basis projections (see [4] for a complete exposition of the concept of a basis projection in the context of CAR-algebras). In the Bose case the Fock state will be characterized by an internal complexification $J$ of the real vector space $h_{\mathrm{B}}$ (see e.g. [7, Section 8.2.4]).

\section{A.4.1. The Fermi case}

Let $\alpha_{g}, g \in \widetilde{\mathfrak{P}_{+}^{\top}}$, denote the Bogoliubov automorphism generated by $V_{\mathrm{F}}(g)$. Then the tupel $\left(\operatorname{CAR}\left(h_{\mathrm{F}}, \Gamma\right), \alpha_{(\cdot)}, \overline{\mathfrak{P}_{+}^{\dagger}}\right)$ is a $\mathrm{C}^{*}$-system [7]. We are interested in a Fock state such that $\alpha_{g}$ is unitarily implementable and such that the unitarities implementing $\alpha_{a}, a \in \mathbb{R}^{4}$, satisfy the spectrality condition (see also [16]).

Define the following operator on $h_{\mathrm{F}}: P(\varphi \oplus \psi):=\varphi \oplus 0$ for all $\varphi \oplus \psi \in h_{\mathrm{F}}$.

\footnotetext{
${ }^{4}$ At this point a simple confirmation of the spin-statistics theorem is possible. Indeed, if onc tries to construct CAR-algebras over $h_{\mathrm{B}}$, the corresponding net will not satisfy the causality property since $n$ is even. Analogous problems with the causality appear if one defines CCR-algebras over $h_{\mathrm{F}}$.

${ }^{5}$ This means, for example, that $\operatorname{spec}_{F}(g)$ is symmetric.
} 
LEMMA A.4.1. $P$ is a basis projection, i.e. $P+\Gamma P \Gamma=\mathbb{1}$, that commutes with $V_{\mathrm{F}}$.

Proof: The statements are direct consequences of the definitions of $P, \Gamma, \Gamma_{1}$ and $V_{\mathrm{F}}$.

The Hilbert space $\mathfrak{H}_{P}$, associated to the representation $\pi_{P}$ characterized by the basis projection $P$, is the antisymmetric part of the Fock space constructed from $P h_{\mathrm{F}} \cong h_{m}$.

THEOREM A.4.2. The automorphisms $\alpha_{g}, g \in \widehat{\mathfrak{P}_{+}^{\uparrow}}$, are unitarily implementable on $\mathfrak{H}_{P}$, i.e. there exists a strongly continuous unitary representation $Q_{(\cdot)}$ of $\widetilde{\mathfrak{P}_{+}^{\uparrow}}$ on $\mathfrak{H}_{P}$ such that

$$
\pi_{P}\left(\alpha_{g}(X)\right)=Q_{g} \pi_{P}(X) Q_{g}^{-1} \quad \text { for all } \quad X \in \operatorname{CAR}\left(h_{\mathrm{F}}, \Gamma\right) .
$$

Furthermore, for all $a \in \mathbb{R}^{4}, Q_{a}$ satisfies the spectrality condition.

Proof: The necessary and sufficient condition for a Bogoliubov automorphism $\alpha_{g}$ generated by $V_{\mathrm{F}}(g)$ to be unitarily implementable is that $P V_{\mathrm{F}}(g)(\mathbb{I}-P)$ belongs to the Hilbert-Schmidt class on $h_{\mathrm{F}^{\prime}}$ (see [4, p. 77]). But in our case $P V_{\mathrm{F}}(g)(\mathbb{I}-P)=0$ and 0 is trivially a Hilbert-Schmidt operator.

Since $V_{\mathrm{F}}$ is a unitary representation of $\widehat{\mathfrak{P}_{+}^{\dagger}}$ that commutes with $\Gamma$ and $P$, it is clear that the associated Bogoliubov automorphisms $\alpha_{g}$ leave the Fock state invariant. (For an explicit expression of the Fock state associated to the basis projection $P$ see [4, Eqs. 2.17 and 2.18].) Therefore, the uniqueness of the GNS-construction yields the uniqueness of $Q_{g}$. But a direct computation shows that the second quantization of $V_{1}(g)$ satifies Eq. (25) on the set of finite particle vectors. Therefore $\left.Q_{9}=\mathbb{I} \oplus \underset{n \geq 1}{\oplus} \stackrel{n}{\otimes} V_{1}(g)\right)$.

For $a \in \mathbb{R}^{4}$, we have that $V_{1}(a)$ satisfies the spectrality condition on the one-particle Hilbert space $h_{m}$. This implies that $Q_{g}$ satisfies the spectrality condition on the Fock space $\mathfrak{H}_{P}$ (see also $[4$, Appendix $\mathrm{C}]$ ).

\section{A.4.2. The Bose case}

As in the preceding section we have $\left(\operatorname{CCR}\left(h_{\mathrm{B}}, \sigma\right), \alpha_{(\cdot)}, \widehat{\mathfrak{P}_{+}^{\top}}\right)$ as a $\mathrm{C}^{*}$-system, where $\alpha_{g}, g \in \widetilde{\mathfrak{P}_{+}^{\dagger}}$, denotes the Bogoliubov automorphisms generated by $V_{\mathrm{B}}(g)$. Again, we are interested in a Fock state where the spectrality condition is satisfied.

LEMMA A.4.3. A real-linear mapping defined by $J\left(\varphi_{1} \oplus \varphi_{2}\right):=\left(i \varphi_{1}\right) \oplus\left(-i \varphi_{2}\right)$ is an internal complexification on $h_{\mathrm{B}}$ (i.e. $J$ satisfies $J^{2}=-\mathbb{I}, \sigma(\varphi, J \psi)=-\sigma(J \varphi, \psi$ ) and $\sigma(\varphi, J \varphi) \geq 0$ for all $\left.\varphi, \psi \in h_{\mathrm{B}}\right)$. J induces a real-valued scalar product $s(\cdot, \cdot)$ and a complex scalar product $k(\cdot, \cdot)$. Finally, the estimate $|\sigma(\varphi, \psi)| \leq\|\varphi\|_{s} \cdot\|\psi\|_{s}$ holds, where $\mid ! \cdot \|_{s}$ denotes a norm induced by $s$.

Proof: The equations $J^{2}=-\mathbb{I}$ and $\sigma(\varphi, J \psi)=-\sigma(J \varphi, \psi)$ are easy to verify. Furthermore, $s(\varphi, \varphi):=\sigma(\varphi, J \varphi)=\left\langle\varphi_{1}, \varphi_{1}\right\rangle_{\beta_{+}}+\left\langle\varphi_{2}, \varphi_{2}\right\rangle_{\beta_{+}} \geq 0$ for all $\varphi=\varphi_{1} \oplus \varphi_{2}$ $\in h_{\mathrm{B}}$. 
From the polarization formula for real scalar products one has $s(\varphi, \psi)=\sigma(\varphi, J \dot{\psi})$ and $k(\varphi, \psi)=s(\varphi, \psi)+i \sigma(\varphi, \psi)=\left\langle\varphi_{1}, \psi_{1}\right\rangle_{\beta_{+}}+\left\langle\psi_{2}, \varphi_{2}\right\rangle_{\beta_{+}}$. (Note that $i k(\varphi, \psi)$ $=k(\varphi, J \psi)$ for all $\varphi, \psi \in h_{\mathrm{B}}$.

Finally, for any $\varphi, \psi \in h_{\mathrm{B}}$ the estimate

$$
|\sigma(\varphi, \psi)| \leq|k(\varphi, \psi)| \leq\|\varphi\|_{k} \cdot\|\psi\|_{k}=\|\varphi\|_{s} \cdot\|\psi\|_{s}
$$

concludes the proof.

REMARK A.4.4: As the complex structure on $h_{\mathrm{B}}$ is completely determined by $J$, one has to adapt to this case the usual definition of infinitesimal generator of a unitary (with respect to $k$ ) group $U(t)$. Concretely, one defines

$$
-J A \varphi:=\lim _{t \rightarrow 0} \frac{U(t) \varphi-\varphi}{t} \quad \text { for all } \varphi \text { such that the limit exists. }
$$

We extend $J$ to the whole symmetric Fock space $\mathcal{F}_{s}\left(h_{\mathrm{B}}\right)$ constructed from $\left(h_{\mathrm{B}}, k\right)$ as usual

$$
\begin{aligned}
& J_{0}:=i, \\
& J_{1}:=J \\
& J_{n}\left(\varphi_{1} \otimes \ldots \otimes \varphi_{n}\right) \\
& \quad:=\left(J \varphi_{1}\right) \otimes \varphi_{2} \otimes \ldots \otimes \varphi_{n}=\ldots=\varphi_{1} \otimes \ldots \otimes \varphi_{n-1} \otimes\left(J \varphi_{n}\right),
\end{aligned}
$$

for all $\varphi_{l} \in h_{\mathrm{B}}, l=1, \ldots, n$. Therefore, $\widehat{J}:=\underset{n \geq 0}{\oplus} J_{n}$ defines a complexification on $\mathcal{F}_{s}\left(h_{\mathrm{B}}\right)$. Note, nevertheless, that in general $(i \varphi) \otimes \psi \neq \varphi \otimes(i \psi), \varphi, \psi \in h_{\mathrm{B}}$, since the construction of the tensor product uses the complex structure given by $J$.

THEOREM A.4.5. The automorphisms $\alpha_{g}, g \in \widehat{\mathfrak{P}_{+}^{\dagger}}$, are unitarily implementable on the Fock space $\mathcal{F}_{s}\left(h_{\mathrm{B}}\right)$ and the unitarities implementing the translation group $\mathbb{R}^{4}$ satisfy the spectrality condition.

Proof: The complexification $J$ defined in Lemma A.4.3 characterizes a Fock state of $\operatorname{CCR}\left(h_{\mathrm{B}}, \sigma\right)$ through the generating functional $\phi(\varphi):=e^{-\frac{1}{4}\|\varphi\|_{s}^{2}}, \varphi \in h_{\mathrm{B}}$. But since $V_{\mathrm{B}}(g)$ is unitary (with respect to $k$ ) on the one-particle Hilbert space $\left(h_{\mathrm{B}}, k\right)$, it is clear that the Bogoliubov automorphisms $\alpha_{g}, g \in \overline{\mathfrak{P}_{+}^{\top}}$, leave the Fock state invariant. The implementability of $\alpha_{g}$ is therefore guaranteed by the uniqueness of the GNS-construction. The unitarities that implement $\alpha_{g}$ are unique.

Denote by $Q_{g}$ the second quantization of $V_{\mathrm{B}}(g)$ on $\mathcal{F}_{s}\left(h_{\mathrm{B}}\right)$. We will prove that

$$
\pi_{J}\left(\alpha_{g}(X)\right)=Q_{g} \pi_{J}(X) Q_{g}^{-1} \quad \text { for all } \quad X \in \operatorname{CCR}\left(h_{\mathrm{B}}, \sigma\right),
$$


where $\pi_{J}$ denotes the Fock representation associated with $J$. It is sufficient to verify Eq. (29) on a total set of $\mathcal{F}_{s}\left(h_{\mathrm{B}}\right)$ for the Weyl elements of $\operatorname{CCR}\left(h_{\mathrm{B}}, \sigma\right)$. But in this case a straightforward calculation shows that Eq. (29) is satisfied on the set of coherent vectors $\left\{\varphi \in \mathcal{F}_{s}\left(h_{\mathrm{B}}\right): \varphi=\left(1, \psi, \frac{1}{\sqrt{2 !}}(\psi \otimes \psi), \ldots\right), \psi \in h_{\mathrm{B}}\right\}$, which is total in $\mathcal{F}_{s}\left(h_{\mathrm{B}}\right)$ (see [17, Chapter 2]).

Finally, taking into account Remark A.4.4, one also has that the infinitesimal generator of the time translation $P_{0}$ and the square of the infinitesimal generator of the spacetime translations $P^{2}$ are positive operators on the one-particle Hilbert space. Concretely, one has $\left(P_{0} \varphi\right)(p)=p_{0} \varphi(p)$ and $\left(P^{2} \varphi\right)(p)=m^{2} \varphi(p)$. This implies that the second quantization of $V_{\mathrm{B}}(g)$ (i.e. $Q_{g}$ ) satisfies the spectrality condition (see $[15, \mathrm{p} .63]$ ).

Part B: The massless case $(m=0)$

\section{B.1. Representation theory for massless models}

The UIR of $\widetilde{\mathfrak{P}_{+}^{\top}}$ can be obtained from the well-known theory of induced representations (see Chapters 2 and 3 of [18] for a neat summary or the standard references $[10],[19]$ and [9]). The strategy is to "induce" the UIR of the whole $\widehat{\mathfrak{P}_{+}^{\dagger}}$ from a finite-dimensional unitary representation of a certain subgroup of the symmetry group $\widehat{\mathfrak{P}_{+}^{\dagger}}$. A characteristic feature of massless models, in contrast to massive ones, is that the corresponding little group is non-compact and therefore certain representations of the little group which are physically motivated have to be selected (see [10, p. 202]). Precisely, the little group of the character $\chi_{q}$ with $q=(1,0,0,1)$ is (see $\left.[20, p .162]\right):^{6}$

$$
\Delta:=\left\{\left(\begin{array}{cc}
e^{\frac{i}{2} \theta} & e^{-\frac{i}{2} \theta} z \\
0 & e^{-\frac{i}{2} \theta}
\end{array}\right) \in \operatorname{SL}(2, \mathbb{C}): \quad \theta \in[0,4 \pi), z \in \mathbb{C}\right\} .
$$

It can also be shown that $\widehat{\mathfrak{P}_{+}^{\uparrow}} / \widetilde{\mathfrak{L}}$ is diffeomorphic to the orbit $\mathcal{C}^{+}:=\left\{p \in \mathbb{R}^{4}: p^{2}\right.$ $\left.=0, p_{0}>0\right\}$, where $\tilde{\mathfrak{L}}:=\Delta(\mathrm{S}) \mathbb{R}^{4}$. Let $\mathfrak{H}:=\mathrm{L}^{2}\left(\mathcal{C}^{+}, \mu_{0}(\mathrm{~d} p)\right)$, where $\mu_{0}(\mathrm{~d} p)=\mathrm{d}^{3} \mathfrak{p} / p_{0}$ is the invariant measure on $\mathcal{C}^{+}$. On $\mathfrak{H}$ we have the following irreducible representation of $\widetilde{\mathfrak{P}_{+}^{\dagger}}$ that satisfies the spectrality condition:

$$
\left(U_{1}(A, a) \varphi\right)(p):=e^{-i p a}\left(\left(H_{p}^{-1} A H_{A_{A}^{-1} p}\right)_{22}\right)^{n} \varphi\left(A_{A}^{-1} p\right)
$$

where $(A, a) \in \widehat{\mathfrak{P}_{+}^{\dagger}}$ and $n / 2$ is the helicity of the model. ${ }^{7}$ The matrices $H_{p} \in \operatorname{SL}(2, \mathbb{C})$ satisfy the equation:

\footnotetext{
${ }^{6}$ The corresponding little group associated to the character $\chi_{q}$ with $q=(m, 0,0,0), m>0$, is $\operatorname{SU}(2)$, which is a compact subgroup of $\operatorname{SL}(2, \mathbb{C})$.

? The representation $U_{1}$ is unitary for the usual $\mathrm{L}^{2}$-scalar product $(\varphi, \psi)=\int_{\mathcal{C}}+\overline{\varphi(p)} \psi(p) \mu .0(\mathrm{~d} p)$.
} 


$$
H_{p}\left(\begin{array}{cc}
2 & 0 \\
0 & 0
\end{array}\right) H_{p}^{*}=P, \text { where } P=\left(\begin{array}{cc}
p_{0}+p_{3} & p_{1}-i p_{2} \\
p_{1}+i p_{2} & p_{0}-p_{3}
\end{array}\right)=\sum_{\mu} p_{\mu} \sigma_{\mu}
$$

by convention $(10)$.

REMARK B.1.1: Notice that the $H_{p}$ matrices are not uniquely determined by Eq. (32), as we can always use $H_{p}^{\prime}=H_{p} L$, with $L \in \Delta$ instead of the original $H_{p}$. Although the matrix $H_{p}^{-1} A H_{A_{A}^{-1} p}$ depends on the choice of the $H_{p}$ matrix, it is easy to see that the matrix elements $\left(H_{p}^{-1} A H_{A_{A}^{-1} p}\right)_{11}$ and $\left(H_{p}^{-1} A H_{A_{A}^{-1} p}\right)_{22}$ do not change if we use $H_{p}^{\prime}$ instead of $H_{p}$. A possible solution of Eq. (32) is

$$
H_{p}=\frac{1}{\sqrt{2 p_{0}\left(p_{0}+p_{3}\right)}}\left(\begin{array}{cc}
-\sqrt{p_{0}}\left(p_{0}+p_{3}\right) & \frac{p_{1}-i p_{2}}{\sqrt{p_{0}}} \\
-\sqrt{p_{0}}\left(p_{1}+i p_{2}\right) & -\frac{p_{0}+p_{3}}{\sqrt{p_{0}}}
\end{array}\right) .
$$

Using the polar decomposition and denoting $p=\left(p_{0}, \mathfrak{p}\right)$ we can write

$$
H_{p}=U^{*}(\mathfrak{p}) H
$$

where

$$
H=\left(\begin{array}{cc}
p_{0}^{\frac{1}{2}} & 0 \\
0 & p_{0}^{-\frac{1}{2}}
\end{array}\right), U(\mathfrak{p})=\frac{1}{\sqrt{2|\mathfrak{p}|\left(|\mathfrak{p}|+p_{3}\right)}}\left(\begin{array}{cc}
-\left(|\mathfrak{p}|+p_{3}\right) & -p_{1}+i p_{2} \\
p_{1}+i p_{2} & -\left(|\mathfrak{p}|+p_{3}\right)
\end{array}\right) .
$$

$H$ represents a boost in the $x_{3}$-direction with the velocity $v_{3}=c\left(p_{0}^{2}-1\right) /\left(p_{0}^{2}+1\right)$, and $U(\mathfrak{p})$ represents a rotation of the reference frame that transforms a 4-vector $p=\left(p_{0}, p_{1}, p_{2}, p_{3}\right)$ into a 4 -vector $\left(p_{0}, 0,0, p_{0}\right)$ (see $[19$, p. 65]).

REMARK B.1.2: For $A=\left(\begin{array}{ll}a & b \\ c & d\end{array}\right), a d-b c=1$, we can compute explicitly

$$
e^{-\frac{2}{2} \theta(A, p)}=\left(H_{p}^{-1} A H_{\Lambda_{A}^{-1} p}\right)_{22}=\frac{-b\left(p_{1}+i p_{2}\right)+d\left(p_{0}+p_{3}\right)}{\left|-b\left(p_{1}+i p_{2}\right)+d\left(p_{0}+p_{3}\right)\right|} .
$$

Standard properties of the map $\theta: \operatorname{SL}(2, \mathbb{C}) \times \mathcal{C}^{+} \rightarrow \mathbb{R}$ are:

(i) $\theta(\mathbb{I I}, p)=\mathbf{0}$.

(ii) $\overline{\theta(A, p)}=\theta(A, p)$.

(iii) $\theta(A B, p)=\theta(A, p)+\theta\left(B, A_{A}^{-1} p\right)$,

where $A, B \in \mathrm{SL}(2, \mathbb{C}), p \in \mathcal{C}^{+}$. The properties (i)-(iii) can be established without knowing the explicit expression of $e^{-\frac{i}{2} \theta(A, p)}$ (see [21]). Furthermore, the following properties are true:

(iv) Let $A:=\left(\begin{array}{ll}a & 0 \\ c & a^{-1}\end{array}\right) \in \operatorname{SL}(2, \mathbb{C})$, with $c \in \mathbb{C}$ and $a>0$. Then $\theta(A, p)=0$ for all $p \in \mathcal{C}^{+}$. In general, for $b=b_{1}+i b_{2}$ and $d=d_{1}+i d_{2}$, 


$$
\begin{aligned}
\operatorname{ker} \theta=\{ & (A, p) \in \operatorname{SL}(2, \mathbb{C}) \times\left(\mathcal{C}^{+} \backslash\{0\}\right): A=\left(\begin{array}{ll}
a & b \\
c & d
\end{array}\right), \\
& \left.\left(p_{0}+p_{3}\right) d_{2}=b_{1} p_{2}+b_{2} p_{1},\left(p_{0}+p_{3}\right) d_{1}>b_{1} p_{1}-b_{2} p_{2}\right\}
\end{aligned}
$$

(v) For all $p_{0}>0, e^{i \theta\left(A,\left(p_{0}, 0,0, \pm p_{0}\right)\right)}=\bar{d} /|d|$. Therefore, $\theta\left(A,\left(p_{0}, 0,0, \pm p_{0}\right)\right)$ does not depend on $p_{0}$. Furthermore, let $A:=\left(\begin{array}{ll}a & 0 \\ c & a^{-1}\end{array}\right) \in \operatorname{SL}(2, \mathbb{C})$, then $e^{i \theta(A, p)}=|a| / \bar{a}$ for all $p \in \mathcal{C}^{+}$.

\section{B.2. The factor spaces $\mathfrak{H}_{ \pm}^{\prime}$ and related topics}

\section{B.2.1. An isometry between two possible representation spaces}

In order to prove the causality and covariance of the net of local $\mathrm{C}^{*}$-algebras, it is more useful to work with the tripel $\left(\mathfrak{H}_{+}^{\prime}, V_{1}^{\prime},\langle\cdot, \cdot\rangle_{\beta_{+}}^{\prime}\right)$, instead of the tripel $\left(\mathfrak{H}, U_{1},(\cdot, \cdot)\right)$ defined in the preceding section. We show that the spaces $\left(\mathfrak{H}_{+}^{\prime},(\cdot, \cdot)_{\beta_{+}}^{\prime}\right)$ and $(\mathfrak{H},(\cdot, \cdot))$ are isometric and that the respective representations are unitarily equivalent. The tripel $\left(\mathfrak{H}_{+}^{\prime}, V_{1}^{\prime},(\cdot, \cdot)_{\beta_{+}}^{\prime}\right)$ will be constructed in this section.

First consider $\mathfrak{H}^{(n)}:=\mathbf{L}^{2}\left(\mathcal{C}^{+}, \mathcal{H}, \mu_{0}(\mathrm{~d} p)\right)$, where $\mathcal{H}$ is the Hilbert space of dimension $n+1$ associated with the spinor representation of $\operatorname{SL}(2, \mathbb{C})$ of type $(n, 0)$ and define the sesquilinear form

$$
\langle\varphi, \psi\rangle_{\beta_{+}}:=\int_{\mathcal{C}^{+}}\left(\varphi(p), \beta_{+}(p) \psi(p)\right)_{\mathcal{H}} \mu_{0}(\mathrm{~d} p), \quad \varphi, \psi \in \mathfrak{H}^{(n)}
$$

with the convention ${ }^{8}$

$$
\beta_{+}(p):=D_{P^{+}}=\bigotimes^{n} P^{+} \quad \text { and } \quad P^{+}=\frac{1}{2}\left(p_{0} \sigma_{0}-\sum_{i} p_{i} \sigma_{i}\right)
$$

Define also the representation $V_{1}$ on $\mathfrak{H}^{(n)}$ by:

$$
\left(V_{1}(g) \varphi\right)(p):=e^{-i p a} D_{A} \varphi\left(\Lambda_{A}^{-1} p\right), \quad g=(A, a) \in \widetilde{\mathfrak{P}_{+}^{\uparrow}},
$$

which leaves the sesquilinear form $\langle\cdot, \cdot\rangle_{\beta_{+}}$invariant.

REMARK B.2.1: Notice that $\operatorname{det} P^{+}=0$, so that $\langle\cdot,\rangle_{\beta_{+}}$is semi-definite ${ }^{9}$ for $n \geq 1$. This confirms a general theorem of Barut and Rączka. As a consequence of the fact that

\footnotetext{
${ }^{8}$ Compare with Eq. (9) for the massive case.

"In standard physical literature expressions like "indefinite form" or "indefinite metric" can also be found.
} 
the little group associated to massless models is solvable, connected and non-compact, the sesquilinear form $\langle,,\rangle_{\beta_{+}}$must necessarily be semi-definite. See [22] for details.

\section{B.2.1.1. The neutrino case $n=1\left(s=\frac{1}{2}\right)$}

LEMMA B.2.2. For $n=1$ (i.e. $\mathcal{H}=\mathbb{C}^{2}$ ) and denoting $\varphi=\left(\varphi_{1}, \varphi_{2}\right) \in \mathfrak{H}_{+}^{(1)}$ we have

(i) $\|\varphi\|_{\beta_{+}}=0$ iff $\left(p_{0}-p_{3}\right) \varphi_{1}(p)=\left(p_{1}-i p_{2}\right) \varphi_{2}(p)$ a.e. for $\mu_{0}(\mathrm{~d} p)$.

(ii) $\mathfrak{H}_{+}^{(1)}=\mathfrak{H}_{+}^{(>)} \oplus \mathfrak{H}_{+}^{(0)}$, where

$$
\begin{gathered}
\mathfrak{H}_{+}^{(>)}=\operatorname{span}\left\{\varphi=\left(\varphi_{1}, \varphi_{2}\right) \in \mathfrak{H}_{+}^{(1)}: \quad\left(p_{0}+p_{3}\right) \varphi_{1}(p)=\left(-p_{1}+i p_{2}\right) \varphi_{2}(p)\right\}, \\
\mathfrak{H}_{+}^{(0)}=\operatorname{span}\left\{\varphi=\left(\varphi_{1}, \varphi_{2}\right) \in \mathfrak{H}_{+}^{(1)}: \quad\left(p_{0}-p_{3}\right) \varphi_{1}(p)=\left(p_{1}-i p_{2}\right) \varphi_{2}(p)\right\}, \\
\mathfrak{H}_{+}^{\prime}:=\mathfrak{H}_{+}^{(1)} / \mathfrak{H}_{+}^{(0)} .
\end{gathered}
$$

Proof: (i) A straightforward calculation shows that

$$
\|\varphi\|_{\beta_{+}}^{2}=\langle\varphi, \varphi\rangle_{\beta_{+}}=\int_{\mathcal{C}^{+}}\left|\sqrt{p_{0}-p_{3}} \varphi_{1}(p)-\frac{p_{1}-i p_{2}}{\sqrt{p_{0}-p_{3}}} \varphi_{2}(p)\right|^{2} \mu_{0}(\mathrm{~d} p) .
$$

(ii) We have

$$
\begin{aligned}
\beta_{+}(p)\left(\begin{array}{c}
\left(-p_{1}+i p_{2}\right) \varphi(p) \\
\left(p_{0}+p_{3}\right) \varphi(p)
\end{array}\right) & =p_{0}\left(\begin{array}{c}
\left(-p_{1}+i p_{2}\right) \varphi(p) \\
\left(p_{0}+p_{3}\right) \varphi(p)
\end{array}\right), \\
\beta_{+}(p)\left(\begin{array}{c}
\left(p_{1}-i p_{2}\right) \varphi(p) \\
\left(p_{0}-p_{3}\right) \varphi(p)
\end{array}\right) & =\left(\begin{array}{l}
0 \\
0
\end{array}\right) .
\end{aligned}
$$

Now, for any $\psi \in \mathfrak{H}_{+}^{(>)}$and any $p \in \mathcal{C}^{+}$the vector $\psi(p) \in \mathcal{H}$ is an eigenvector of $\beta_{+}(p)$ with eigenvalue $p_{0}$. The elements of $\mathfrak{H}_{+}^{(0)}$ are eigenvectors with eigenvalue 0 . We can decompose a vector of $\mathfrak{H}_{+}^{(1)}$ in a unique way into a direct sum of vectors of $\mathfrak{H}_{+}^{(>)}$and $\mathfrak{H}_{+}^{(0)}$.

LEMMA B.2.3. Using the preceding notation the following statements are true:

(i) $\mathfrak{H}_{+}^{(0)}$ is $V_{1}$-invariant.

(ii) $V_{1}$ leaves the $\|\cdot\|_{\beta_{+}}$-norm of the elements in $\mathfrak{H}_{+}^{(>)}$invariant, but the space $\mathfrak{H}_{+}^{(>)}$itself is not $V_{1}$-invariant.

(iii) In each coset ${ }^{10}[\varphi]_{+} \in \mathfrak{H}_{+}^{\prime}$ there exists a unique representative $\varphi^{(>)} \in[\varphi]_{+}$ with $\varphi^{(>)} \in \mathfrak{H}_{+}^{(>)}$.

\footnotetext{
${ }^{10}$ The bracket []$_{+}$denotes the equivalence class with respect to the semi-definite sesquilinear form $\langle\cdot,\rangle_{\beta_{+}}$.
} 
Proof: (i) This follows from the fact that $V_{1}$ leaves $\langle\cdot, \cdot\rangle_{\beta_{+}}$invariant. This statement can be also confirmed explicitly: if we define $\alpha(p):=\left(p_{1}-i p_{2}\right) /\left(p_{0}-p_{3}\right)$ and $p^{\prime}$ $:=\Lambda_{A}^{-1} p$, then for $\varphi(p)=\left(\begin{array}{c}\alpha(p) \varphi_{1}(p) \\ \varphi_{1}(p)\end{array}\right)$ we have

$$
\psi(p):=\left(V_{1}(g) \varphi\right)(p)=\left(\begin{array}{c}
\alpha(p) \psi_{1}(p) \\
\psi_{1}(p)
\end{array}\right)
$$

with $\psi_{1}(p)=e^{-i p a}\left(c \alpha\left(p^{\prime}\right)+d\right) \varphi_{1}\left(p^{\prime}\right)$ and where we have used the relation $\alpha(p)$ $=\left(a \alpha\left(p^{\prime}\right)+b\right) /\left(c \alpha\left(p^{\prime}\right)+d\right)$.

(ii) Define $\psi:=V_{1}(g) \varphi, \varphi \in \mathfrak{H}_{+}^{(>)}$and $g \in \widetilde{\mathfrak{P}_{+}^{\dagger}}$, and confirm that $\beta(p) \psi(p)$ $\neq p_{0} \psi(p)$. This shows that, although $\|\psi\|_{\beta_{+}}=\| \varphi ! \beta_{+}$, the function $\psi$ must be generally written as $\psi=\psi^{(>)} \oplus \psi^{(0)}$ with $\psi^{(>)} \in \mathfrak{H}_{+}^{(>)}$and $\psi^{(0)} \in \mathfrak{H}_{+}^{(0)}$.

(iii) is a consequence of Lemma B.2.2 (ii).

DEFINITION B.2.4. We define on $\mathfrak{H}_{+}^{\prime}$ a representation $V_{1}^{\prime}$ of $\widetilde{\mathfrak{P}_{+}^{\top}}$ and the sesquilinear form $\langle\cdot, \cdot\rangle_{\beta_{+}}$by

$$
\begin{gathered}
V_{1}^{\prime}(g)[\varphi]_{+}:=\left[V_{1}(g) \varphi\right]_{+} \\
\left\langle[\varphi]_{+},[\psi]_{+}\right\rangle_{\beta_{+}}^{\prime}:=\int_{\mathcal{C}^{+}}\left(\varphi(p), \beta_{+}(p) \psi(p)\right)_{\mathcal{H}} \mu_{0}(\mathrm{~d} p) .
\end{gathered}
$$

REMARK B.2.5: $V_{1}^{\prime}$ and $\langle\cdot, \cdot\rangle_{\beta_{+}}^{\prime}$ are well defined (i.e. they do not depend on representatives of the equivalence class). Furthermore, $\langle\cdot, \cdot\rangle_{\beta_{+}}^{\prime}$ defines a scalar product on $\mathfrak{H}_{+}^{\prime}$ with respect to which $V_{1}^{\prime}$ is unitary. Indeed, from Lemma B.2.3 (ii) it follows that $\left[V_{1}(g) \varphi\right]_{+}=\left[V_{1}(g)\left(\varphi+\varphi^{(0)}\right)\right]_{+}$for any $\varphi^{(0)} \in \mathfrak{H}_{+}^{(0)}$. The equation

$$
\left(\varphi(p)+\varphi^{(0)}(p), \beta_{+}(p)\left(\psi(p)+\psi^{(0)}(p)\right)\right)_{\mathcal{H}}=\left(\varphi(p), \beta_{+}(p) \psi(p)\right)_{\mathcal{H}}
$$

also holds for any $\psi^{(0)}, \varphi^{(0)} \in \mathfrak{H}_{+}^{(0)}$, so that $\langle\cdot, \cdot\rangle_{\beta_{+}}^{\prime}$ is well defined.

THEOREM B.2.6. For $n=1$ a linear map $\Phi_{+}:\left(\mathfrak{F}, U_{1},(\cdot, \cdot)\right) \rightarrow\left(\mathfrak{H}_{+}^{\prime}, V_{1}^{\prime},\langle\cdot, \cdot\rangle_{\beta_{+}}^{\prime}\right)$, defined by $\left(\Phi_{+} \varphi\right)(p):=\left[H_{p}\left(\begin{array}{c}0 \\ \varphi(p)\end{array}\right)\right]_{+}, \varphi \in \mathfrak{H}$, is an isometry that commutes with the representations (i.e. $\Phi_{+} U_{1}=V_{1}^{\prime} \Phi_{+}$).

Proof: Notice that

$$
H_{p}\left(\begin{array}{c}
0 \\
\varphi(p)
\end{array}\right)=-\frac{1}{\sqrt{2 p_{0}^{2}\left(p_{0}+p_{3}\right)}}\left(\begin{array}{c}
\left(-p_{1}+i p_{2}\right) \varphi(p) \\
\left(p_{0}+p_{3}\right) \varphi(p)
\end{array}\right)
$$


is the representative of $\left[H_{p}\left(\begin{array}{c}0 \\ \varphi(p)\end{array}\right)\right]_{+}$in $\mathfrak{H}_{+}^{(>)}$. It is easy to see that $\Phi_{+}$is a bijection between $\mathfrak{h}$ and $\mathfrak{H}_{+}^{\prime}$. Furthermore,

$$
\left\langle\Phi_{+} \varphi_{1}, \Phi_{+} \varphi_{2}\right\rangle_{\beta_{+}}^{\prime}=\left(\varphi_{1}, \varphi_{2}\right)_{\mathrm{L}^{2}}
$$

where we used the relation $\beta_{+}(p)=\left(H_{p}^{-1}\right)^{*}\left(\begin{array}{ll}0 & 0 \\ 0 & 1\end{array}\right) H_{p}^{-1}$.

The proof that $\Phi_{+}$intertwines between $U_{1}$ and $V_{1}^{\prime}$ is straightforward. Let $g$ $=(A, a) \in \widehat{\mathfrak{P}_{+}^{\uparrow}}$

$$
\left(\Phi_{+} U_{1}(g) \varphi\right)(p)=\left[e^{-i p a}\left(H_{p}^{-1} A H_{A_{A}^{-1} p}\right)_{22} H_{p}\left(\begin{array}{c}
0 \\
\varphi\left(A_{A}^{-1} p\right.
\end{array}\right)\right]_{+}
$$

and further

$$
\begin{aligned}
\left(V_{1}^{\prime}(g) \Phi_{+} \varphi\right)(p) & =\left[e^{-i p a} H_{p}\left(H_{p}^{-1} A H_{A_{A}^{-1} p}\right)\left(\begin{array}{c}
0 \\
\varphi\left(\Lambda_{A}^{-1} p\right)
\end{array}\right)\right]_{+} \\
& =\left[e^{-i p a}\left(H_{p}^{-1} A H_{\Lambda_{A}^{-1} p}\right)_{22} H_{p}\left(\begin{array}{c}
0 \\
\varphi\left(\Lambda_{A}^{-1} p\right)
\end{array}\right)\right]_{+},
\end{aligned}
$$

where we used $H_{(\cdot)}\left(\begin{array}{c}\psi(\cdot) \\ 0\end{array}\right) \in \mathfrak{H}_{+}^{(0)}$ for all $\psi \in \mathfrak{H}$.

COROLlaRY B.2.7. $V_{1}^{\prime}$ acts irreducibly on $\mathfrak{H}_{+}^{\prime}$.

\section{B.2.1.2. The case of arbitrary helicity}

The image of $\varphi \in \mathfrak{H}_{+}^{(n)}$ is of the form $\varphi(p)=\operatorname{Sym}\left(\varphi_{1}(p) \otimes \cdots \otimes \varphi_{n}(p)\right)$, where $\varphi_{l} \in \mathfrak{H}_{+}^{(1)}$ for $l=1, \ldots, n$, and Sym is the symmetrization operator on $\otimes \mathrm{C}^{2}$. From Lemma B.2.3 (iii) we can decompose the image of each $\varphi_{l} \in \mathfrak{H}_{+}^{(1)}$ in

$$
\varphi_{l}(p)=\left(\begin{array}{c}
-p_{1}+i p_{2} \\
p_{0}+p_{3}
\end{array}\right) \alpha_{l}(p)+\left(\begin{array}{c}
p_{1}-i p_{2} \\
p_{0}-p_{3}
\end{array}\right) \beta_{l}(p), \quad l=1, \ldots, n .
$$

Therefore,

$$
\varphi(p)=\bigotimes\left(\begin{array}{c}
-p_{1}+i p_{2} \\
p_{0}+p_{3}
\end{array}\right) \alpha_{1}(p) \cdots \alpha_{n}(p)+R\left(\alpha_{k}, \beta_{l}, p\right)
$$

where $\left\|R\left(\alpha_{k}, \beta_{l}, p\right)\right\|_{\beta_{+}}=0$ (see Eq. (38)). The preceding lines motivate the following definition:

DEFINITION B.2.8. Let $\langle\cdot,\rangle_{\beta_{+}}$be the sesquilinear form defined in Eq. (38)

$$
\mathfrak{H}_{+, n}^{(0)}:=\operatorname{span}\left\{\varphi \in \mathfrak{H}_{+}^{(n)}:\langle\varphi, \varphi\rangle_{B_{+}}=0\right\},
$$




$$
\begin{gathered}
\mathfrak{H}_{+, n}^{(>)}:=\operatorname{span}\left\{\varphi \in \mathfrak{H}_{+}^{(n)}: \varphi(p)=\bigotimes^{n}\left(\begin{array}{c}
-p_{1}+i p_{2} \\
p_{0}+p_{3}
\end{array}\right) \chi(p), \text { for some function } \chi\right\}, \\
\mathfrak{H}_{+, n}^{\prime}:=\mathfrak{H}_{+}^{(n)} / \mathfrak{H}_{+, n}^{(0)} .
\end{gathered}
$$

REMARK B.2.9: Note that:

(i) For $n=1$ we recover the definitions (41), (42) and (43), respectively.

(ii) As in the case $n=1$ we can find in each coset $[\varphi]_{+} \in \mathfrak{H}_{+, n}^{\prime}$ a unique representative $\varphi^{(>)} \in[\varphi]_{+}$with $\varphi^{(>)} \in \mathfrak{H}_{+, n}^{(>)}$. We have $\mathfrak{H}_{+}^{(n)}=\mathfrak{H}_{+, n}^{(>)} \ominus \mathfrak{H}_{+, n}^{(0)}$.

LEMMA B.2.10. Let $V_{1}$ be defined as in (40). Then $\mathfrak{H}_{+, n}^{(0)}$ is $V_{1}$-invariant, while $\mathfrak{H}_{+, n}^{(>)}$ is not $V_{1}$-invariant.

Proof: Apply Lemma B.2.3 to each component of the tensor product.

DEFINITION B.2.11. We define on $\mathfrak{H}_{+, n}^{\prime}$ representations $V_{1}^{\prime}$ of $\widetilde{\mathfrak{P}_{+}^{\top}}$ and a sesquilinear form $\langle\cdot, \cdot\rangle_{\beta_{+}}^{\prime}$ :

$$
\begin{aligned}
V_{1}^{\prime}(g)[\varphi]_{+} & :=\left[V_{1}(g) \varphi\right]_{+}, \\
\left\langle[\varphi]_{+},[\psi]_{+}\right\rangle_{\beta_{+}}^{\prime} & :=\int_{\mathcal{C}^{+}}\left(\varphi(p), \beta_{+}(p) \psi(p)\right)_{\mathcal{H}} \mu_{0}(\mathrm{~d} p) .
\end{aligned}
$$

REMARK B.2.12: Using the same arguments as in Remark B.2.5, for each component of the tensor product it is easy to see that $V_{1}^{\prime}$ and $\langle\cdot, \cdot\rangle_{\beta_{+}}^{\prime}$ are well defined.

THEOREM B.2.13. A linear map $\Phi_{+}:\left(\mathfrak{H}, U_{1},(\cdot, \cdot)\right) \rightarrow\left(\mathfrak{H}_{+, n}^{\prime}, V_{1}^{\prime},\langle\cdot, \cdot\rangle_{\beta_{+}}^{\prime}\right)$ defined by

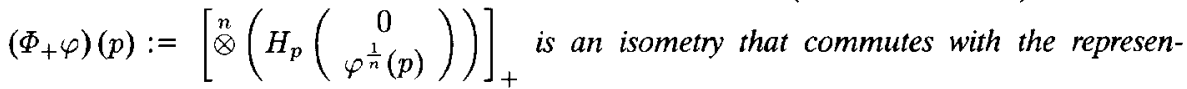
tations (i.e. $\left.\Phi_{+} U_{1}=V_{1}^{\prime} \Phi_{+}\right)$.

Proof: Although $\varphi^{\frac{1}{n}}$ is a multi-valued function, any determination of $\varphi^{\frac{1}{n}}$ yields the same value of $\left(\Phi_{+} \varphi\right)(p)$.

$$
\bigotimes^{n}\left(H_{p}\left(\begin{array}{c}
0 \\
\varphi^{\frac{1}{n}}(p)
\end{array}\right)\right)=\left(\bigotimes^{n}\left(-\frac{1}{\sqrt{2 p_{0}^{2}\left(p_{0}+p_{3}\right)}}\left(\begin{array}{c}
-p_{1}+i p_{2} \\
p_{0}+p_{3}
\end{array}\right)\right)\right) \varphi(p)
$$

is a representative of $\Phi_{+} \varphi \in \mathfrak{H}_{+, n}^{\prime}$ in $\mathfrak{H}_{+, n}^{(>)} . \Phi_{+}$is therefore bijective.

Finally, for $g=(A, a) \in \widetilde{\mathfrak{P}_{+}^{\dagger}}$ and $\varphi \in \mathfrak{H}$, 


$$
\begin{aligned}
& \left(\Phi_{+} U_{1}(g) \varphi\right)(p)=\left[e^{-i p a}\left(H_{p}^{-1} A H_{A_{A}^{-1} p}\right)_{22}^{n} D_{H_{p}}\left(\bigotimes\left(\begin{array}{c}
0 \\
\varphi^{\frac{1}{n}}\left(A_{A}^{-1} p\right)
\end{array}\right)\right)\right]_{+}, \\
& \left(V_{\mathrm{I}}^{\prime}(g) \Phi_{+} \varphi\right)(p)=\left[e^{-i p a} D_{H_{p}} D_{H_{p}^{-1}} D_{A} D_{H_{A}^{-1}} \bigotimes^{n}\left(\begin{array}{c}
0 \\
\varphi^{\frac{1}{n}}\left(A_{A}^{-1} p\right)
\end{array}\right)\right]_{+} \\
& =\left[e^{-i p a}\left(H_{p}^{-1} A H_{A_{A}^{-1} p}\right)_{22}^{n} D_{H_{p}}\left(\bigotimes^{n}\left(\begin{array}{c}
0 \\
\varphi^{\frac{1}{n}}\left(A_{A}^{-1} p\right)
\end{array}\right)\right)\right]_{+} \text {, }
\end{aligned}
$$

where for the last equation we have used $\stackrel{n}{\otimes}\left(H_{(\cdot)}\left(\begin{array}{c}\psi(\cdot) \\ 0\end{array}\right)\right) \in \mathfrak{H}_{+, n}^{(0)}$ for all $\psi \in \mathfrak{H}$.

COROLlaRY B.2.14. $V_{1}^{\prime}$ acts irreducibly on $\mathfrak{H}_{+, n}^{\prime}$.

\section{B.2.2. Analogous arguments for models with opposite helicity}

For the construction of the net of linear submanifolds in the reference space of the CAR- and CCR-algebras, respectively, the representation associated to models with opposite helicity is also necessary. For the convenience of the reader we will list in this case the analogous definitions and results of the preceding section. (The index 1 will be replaced by the index 4.) The choice of the index 4 for this representation will be justified in Section B.3.1.

On the space $\mathfrak{H}$ we define in this section the representation

$$
\left(U_{4}(g) \varphi\right)(p):=e^{-i p a} e^{+\frac{i}{2} \theta(A, p)} \varphi\left(A_{A}^{-1} p\right)
$$

(see Eq. (36)). Further, we consider the tripel $\left(\mathfrak{H}_{-}^{(n)}, V_{4},\langle\cdot,\rangle_{\beta_{-}}\right)$:

$$
\langle\varphi, \psi\rangle_{\beta_{-}}:=\int_{\mathcal{C}^{+}}\left(\varphi(p), \beta_{-}(p) \psi(p)\right)_{\mathcal{H}} \mu_{0}(\mathrm{~d} p),
$$

where $\varphi, \psi \in \mathfrak{H}_{-}^{(n)}, \beta_{-}(p):=\Gamma_{0} \beta_{+}(p) \Gamma_{0}$ and $\Gamma_{0}$ denotes an anti-unitary involution on the space $\mathcal{H}$. Finally, let $V_{\mathbf{4}}$ be a representation of $\widetilde{\mathfrak{P}_{+}^{\top}}$ over $\mathfrak{H}_{-}^{(n)}$ defined by

$$
\left(V_{4}(g) \varphi\right)(p):=e^{-i p a} D_{\bar{A}} \varphi\left(\Lambda_{A}^{-1} p\right) .
$$

For $n=1$ we have the following results.

LEMMA B.2.15. Let $\varphi=\left(\varphi_{1}, \varphi_{2}\right) \in \mathfrak{H}_{-}^{(1)}$. Then

(i) $\|\varphi\|_{\beta_{-}}=0$ iff $\left(p_{0}-p_{3}\right) \varphi_{1}(p)=\left(p_{1}+i p_{2}\right) \varphi_{2}(p)$ a.e. for $\mu_{0}(\mathrm{~d} p)$.

(ii) $\mathfrak{H}_{-}^{(1)}=\mathfrak{H}_{-}^{(>)} \oplus \mathfrak{H}_{-}^{(0)}$, where 


$$
\begin{gathered}
\mathfrak{H}_{-}^{(>)}=\operatorname{span}\left\{\varphi=\left(\varphi_{1}, \varphi_{2}\right) \in \mathfrak{H}_{-}^{(1)}: \quad\left(p_{0}+p_{3}\right) \varphi_{1}(p)=-\left(p_{1}+i p_{2}\right) \varphi_{2}(p)\right\} \\
\mathfrak{H}_{-}^{(0)}=\operatorname{span}\left\{\varphi=\left(\varphi_{1}, \varphi_{2}\right) \in \mathfrak{H}_{-}^{(1)}: \quad\left(p_{0}-p_{3}\right) \varphi_{1}(p)=\left(p_{1}+i p_{2}\right) \varphi_{2}(p)\right\} \\
\mathfrak{H}_{-}^{\prime}:=\mathfrak{H}_{-}^{(1)} / \mathfrak{H}_{-}^{(0)} .
\end{gathered}
$$

DEFINITION B.2.16. Using similar arguments as in Definition B.2.4 and Remark B.2.5 we define on $\mathfrak{H}_{-}^{\prime}$ a representation $V_{4}^{\prime}$ of $\widetilde{\mathfrak{P}_{+}^{\dagger}}$ and a sesquilinear form $\langle\cdot, \cdot)_{\beta_{-}}^{\prime}$ by ${ }^{11}$

$$
\begin{gathered}
V_{4}^{\prime}(g)[\varphi]_{-}:=\left[V_{4}(g) \varphi\right]_{-}, \\
\left\langle[\varphi]_{-},[\psi]_{-}\right\rangle_{\beta_{-}}^{\prime}:=\int_{\mathcal{C}^{+}}\left(\varphi(p), \beta_{-}(p) \psi(p)\right)_{\mathcal{H}} \mu_{0}(\mathrm{~d} p) .
\end{gathered}
$$

We conclude the case $n=1$ with the following theorem:

THEOREM B.2.17. For $n=1$ a linear map $\Phi_{--}:\left(H_{H}, U_{4},(\cdot, \cdot)\right) \rightarrow\left(\mathfrak{H}_{-}^{\prime}, V_{4}^{\prime},\langle\cdot, \cdot\rangle_{\beta_{-}}^{\prime}\right)$ defined by $\left(\Phi_{-} \varphi\right)(p):=\left[\Gamma_{0} H_{p} \Gamma_{0}\left(\begin{array}{c}0 \\ \varphi(p)\end{array}\right)\right], \varphi \in \mathfrak{H}$, is an isometry that commutes with the representations (i.e. $\Phi_{-} U_{4}=V_{4}^{\prime} \Phi_{-}$).

In the general case we define

DEFINITION B.2.18. (See analogous statements in Definition B.2.8 and Remark B.2.9.)

$$
\begin{gathered}
\mathfrak{H}_{-, n}^{(0)}:=\operatorname{span}\left\{\varphi \in \mathfrak{H}_{-}^{(n)}:\langle\varphi, \varphi\rangle_{\beta_{-}}=0\right\} \\
\mathfrak{H}_{-, n}^{(>)}:=\operatorname{span}\left\{\rho \in \mathfrak{H}_{-}^{(n)}: \varphi(p)=\bigotimes^{n}\left(\begin{array}{c}
p_{1}+i p_{2} \\
-\left(p_{0}+p_{3}\right)
\end{array}\right) \chi(p), \text { for some function } \chi\right\} \\
\mathfrak{H}_{-, n}^{\prime}:=\mathfrak{H}_{-}^{(n)} / \mathfrak{H}_{-, n}^{(0)} .
\end{gathered}
$$

DEFINITION B.2.19. On $\mathfrak{H}_{-, n}^{\prime}$ we define representations $V_{4}^{\prime}$ of $\widetilde{\mathfrak{P}_{+}^{\dagger}}$ and a sesquilinear form $\langle\cdot, \cdot\rangle_{\beta_{-}}^{\prime}$ as

$$
\begin{gathered}
V_{4}^{\prime}(g)[\varphi]_{-}:=\left[V_{4}(g) \varphi\right]_{-}, \\
\left\langle[\varphi]_{-},[\psi]_{-}\right\rangle_{\beta_{-}}^{\prime}:=\int_{\mathcal{C}^{+}}\left(\varphi(p), \beta_{-}(p) \psi(p)\right)_{\mathcal{H}} \mu_{0}(\mathrm{~d} p) .
\end{gathered}
$$

\footnotetext{
${ }^{11}$ The bracket $[\cdot]_{-}$denotes the equivalence class with respect to the semi-definite sesquilinear form $\langle\cdot, \cdot\rangle_{B_{-}} \cdot$
} 
The following theorem concludes the section.

THEOREM B.2.20. The linear map $\Phi_{-}:\left(\mathfrak{H}, U_{4},(\cdot, \cdot)\right) \rightarrow\left(\mathfrak{s}_{-, n}^{\prime}, V_{4}^{\prime},\langle\cdot, \cdot\rangle_{\beta_{-}}^{\prime}\right)$ defined by $\left(\Phi_{-} \varphi\right)(p):=\left[\otimes\left(\Gamma_{0} H_{p} \Gamma_{0}\left(\begin{array}{c}0 \\ \varphi^{\frac{1}{n}}(p)\end{array}\right)\right)\right]$ is an isometry that commutes with the representations (i.e. $\Phi_{-} U_{4}=V_{4}^{\prime} \Phi_{-}$).

\section{B.3. Causal and covariant nets of local $\mathrm{C}^{*}$-algebras}

In this section we will define the reference spaces $h_{\mathrm{F}}$ and $h_{\mathrm{B}}$. In order to construct causal and covariant nets of local $\mathrm{C}^{*}$-subalgebras of the algebras $\operatorname{CAR}\left(h_{\mathrm{F}}, \Gamma\right)$ and $\operatorname{CCR}\left(h_{\mathrm{B}}, \sigma\right)$, it is sufficient to specify nets of linear submanifolds in the corresponding reference spaces that satisfy the properties $(0)_{\mathrm{F}}-(\text { III })_{\mathrm{F}}$ and $(\mathrm{I})_{\mathrm{B}}-(\mathrm{III})_{\mathrm{B}}$ of Section 2 , respectively.

In the following $\mathcal{O}, \mathcal{O}_{1}$ and $\mathcal{O}_{2}$ denote open and bounded regions of the Minkowski space.

\section{B.3.1. Representations of $\overline{\mathfrak{P}_{+}^{\top}}$}

Consider on $\mathrm{C}_{0}^{x}\left(\mathbb{R}^{4}, \mathcal{H}\right)$ the following representations of $\overline{\mathfrak{P}_{+}^{\top}}$. We define for $f$ $\in \mathbb{C}_{0}^{x}\left(\mathbb{R}^{4}, \mathcal{H}\right)$ and $g=(A, a) \in \widetilde{\mathfrak{P}_{+}^{\dagger}}$

$$
\begin{aligned}
& \left(T_{1}(g) f\right)(x):=D_{A} f\left(A_{A}^{-1}(x-a)\right), \\
& \left(T_{2}(g) f\right)(x):=D_{\bar{A}} f\left({A_{A}}^{1}(x-a)\right) .
\end{aligned}
$$

Define then $T(g):=T_{1}(g) \oplus T_{2}(g)$, which is a representation of $\mathfrak{P}_{+}^{\top}$ on $\mathrm{C}_{0}^{\infty}\left(\mathbb{R}^{4}, \mathcal{H}\right) \oplus$ $\mathrm{C}_{0}^{\infty}\left(\mathbb{R}^{4}, \mathcal{H}\right)$.

Furthermore, define the following unitary strongly continuous representations on $\mathfrak{H}_{+, n}^{\prime}$ or $\mathfrak{H}_{-, n}^{\prime}$ :

$$
\begin{aligned}
& \left(V_{1}^{\prime}(g)[\varphi]_{+}\right)(p):=\left[e^{-i p a} D_{A} \varphi\left(A_{A}^{-1} p\right)\right]_{+}, \\
& \left(V_{2}^{\prime}(g)[\varphi]_{+}\right)(p):=\left[e^{i p a} D_{A} \varphi\left(A_{A}^{-1} p\right)\right]_{+}, \\
& \left(V_{3}^{\prime}(g)[\varphi]_{-}\right)(p):=\left[e^{i p a} D_{\bar{A}} \varphi\left(A_{A}^{-1} p\right)\right]_{-}, \\
& \left(V_{4}^{\prime}(g)[\varphi]_{-}\right)(p):=\left[e^{-i p a} D_{\bar{A}} \varphi\left(A_{A}^{-1} p\right)\right]_{-} .
\end{aligned}
$$

where $g \subset \overline{\mathfrak{P}_{+}^{\dagger}}$ and $[\varphi]_{ \pm} \in \mathfrak{H}_{ \pm, n}^{\prime}$. 


\section{B.3.2. The Fermi case ( $n$ odd)}

\section{B.3.2.1. CAR-algebras}

We consider the complex Hilbert space $h_{\mathrm{F}}:=\mathfrak{H}_{+, n}^{\prime} \oplus \mathfrak{H}_{+, n}^{\prime} \oplus \mathfrak{H}_{-, n}^{\prime} \oplus \mathfrak{H}_{-, n}^{\prime}$ with a scalar product defined by

$$
\begin{aligned}
\langle[\varphi],[\psi]\rangle_{\mathrm{F}}:= & \left\langle\left[\varphi_{1}\right]_{+},\left[\psi_{1}\right]_{+}\right\rangle_{\beta_{+}}^{\prime}+\left\langle\left[\varphi_{2}\right]_{+},\left[\psi_{2}\right]_{+}\right\rangle_{\beta_{+}}^{\prime}+ \\
& +\left\langle\left[\varphi_{3}\right]_{-},\left[\psi_{3}\right]_{-}\right\rangle_{\beta_{-}}^{\prime}+\left\langle\left[\varphi_{4}\right]_{-},\left[\psi_{4}\right]_{-}\right\rangle_{\beta_{-}}^{\prime}
\end{aligned}
$$

for all $[\varphi]=\left[\varphi_{1}\right]_{+} \oplus\left[\varphi_{2}\right]_{+} \oplus\left[\varphi_{3}\right]_{-} \oplus\left[\varphi_{4}\right]_{-},[\psi]=\left[\psi_{1}\right]_{+} \oplus\left[\psi_{2}\right]_{+} \oplus\left[\psi_{3}\right]_{-} \oplus\left[\psi_{4}\right]_{-} \in h_{\mathrm{F}}$. As anti-unitary involution we use

$$
\Gamma\left(\left[\varphi_{1}\right]_{+} \oplus\left[\varphi_{2}\right]_{+} \oplus\left[\varphi_{3}\right]_{-} \oplus\left[\varphi_{4}\right]_{-}\right):=\left[\Gamma_{0} \varphi_{3}\right]_{+} \oplus\left[\Gamma_{0} \varphi_{1}\right]_{+} \oplus\left[\Gamma_{0} \varphi_{1}\right]_{-} \oplus\left[\Gamma_{0} \varphi_{2}\right]_{-} .
$$

Note that the definition of $\Gamma$ is consistent with the specification of the equivalence classes $[\cdot]_{+}$and $[\cdot]_{-} . \operatorname{CAR}\left(h_{\mathrm{F}}, \Gamma\right)$ is then given uniquely.

\section{B.3.2.2. Existence theorems for the local algebras}

Using the results of Section B.3.1 we have that the definition

$$
V_{\mathrm{F}}(g):=V_{1}^{\prime}(g) \oplus V_{2}^{\prime}(g) \oplus V_{3}^{\prime}(g) \oplus V_{4}^{\prime}(g)
$$

specifies a reducible, unitary, strongly continuous representation of $\overline{\mathfrak{P}_{+}}$over $h_{\mathrm{F}}$.

LEMMA B.3.1. $\Gamma$ and $V_{\mathrm{F}}$ commute (i.e. $\Gamma V_{\mathrm{F}}(g)=V_{\mathrm{F}}(g) \Gamma$ for all $\left.g \in \widehat{\mathfrak{P}_{+}^{\dagger}}\right)$.

Proof: The statement of the Lemma is a direct consequence of the definitions of $V_{\mathbf{F}}$ and $\Gamma$.

In order to relate the test functions over "position-space" with elements of the reference space consider the embedding $\mathfrak{I}_{\mathrm{F}}: \mathrm{C}_{0}^{\infty}\left(\mathbb{R}^{4}, \mathcal{H}\right) \oplus \mathrm{C}_{0}^{\infty}\left(\mathbb{R}^{4}, \mathcal{H}\right) \rightarrow h_{\mathrm{F}}$, defined by

$$
\left(\mathfrak{I}_{\mathrm{F}}\left(f_{1} \oplus f_{2}\right)\right)(p):=\left[\left(\mathfrak{I}_{+} f_{1}\right)\right]_{+} \oplus\left[\left(\mathfrak{I}_{-} f_{1}\right)\right]_{+} \oplus\left[\left(\mathfrak{I}_{-} f_{2}\right)\right]_{-} \oplus\left[\left(\mathfrak{I}_{+} f_{2}\right)\right]_{-},
$$

for all $f_{1} \oplus f_{2} \in \mathrm{C}_{0}^{\infty}\left(\mathbb{R}^{4}, \mathcal{H}\right) \oplus \mathrm{C}_{0}^{\infty}\left(\mathbb{R}^{4}, \mathcal{H}\right)$, and for $\left(\mathfrak{I}_{ \pm} f\right)(p):=\widehat{f}( \pm p){ }^{12}$

\footnotetext{
${ }^{12}$ The hat ${ }^{-}$means the Fourter transformation restricted to the forward light cone $\mathcal{C}^{+}$(compare also with Corollary A.1.3 for the massive case).
} 
The covariance of the net will be based on the following result:

LEMMA B.3.2. The embedding $\mathfrak{I}_{\mathrm{F}}$ intertwines between the representations $T$ and $V_{\mathrm{F}}$ (i.e. $\mathfrak{I}_{\mathrm{F}} T(g)=V_{\mathrm{F}}(g) \mathfrak{I}_{\mathrm{F}}$ for all $g \in \widehat{\mathfrak{P}_{+}^{\top}}$ ).

Proof: Apply the definitions of $T, V_{\mathrm{F}}$ and $\Im_{\mathrm{F}}$ and for all $f \in \mathrm{C}_{0}^{\infty}\left(\mathbb{R}^{4}, \mathcal{H}\right)$ use the relations:

$$
\begin{array}{ll}
{\left[\mathfrak{J}_{+}\left(T_{1}(g) f\right)\right]_{+}=V_{1}^{\prime}(g)\left[\mathfrak{I}_{+} f\right]_{+},} & {\left[\mathfrak{I}_{-}\left(T_{1}(g) f\right)\right]_{+}=V_{2}^{\prime}(g)\left[\mathfrak{I}_{-} f\right]_{+}} \\
{\left[\mathfrak{I}_{-}\left(T_{2}(g) f\right)\right]_{-}=V_{3}^{\prime}(g)\left[\mathfrak{I}_{-} f\right] .} & {\left[\mathfrak{I}_{+}\left(T_{2}(g) f\right)\right]_{-}=V_{\mathbf{1}}(g)\left[\mathfrak{I}_{+} f\right]_{-}}
\end{array}
$$

Define the net of linear submanifolds of $h_{\mathrm{F}}$ by

$$
\begin{aligned}
\mathbb{R}^{4} & \supset \mathcal{O} \longmapsto M_{\mathrm{F}}(\mathcal{O}) \\
& =\operatorname{span}\left\{\Im_{\mathrm{F}}\left(f_{1} \oplus f_{2}\right): f_{i} \in \mathrm{C}_{0}{ }^{\mathrm{x}}\left(\mathbb{R}^{4}, \mathcal{H}\right), \operatorname{supp} f_{i} \subset \mathcal{O}, i=1,2\right\} .
\end{aligned}
$$

THEORFM B.3.3. The net $\mathcal{O} \mapsto M_{\mathrm{F}}(\mathcal{O})$ satisfies the properties $(0)_{\mathrm{F}},(\mathrm{I})_{\mathrm{F}},(\mathrm{II})_{\mathrm{F}}$ and (III) $\mathrm{r}$ of Section 2.

Proof: The $\Gamma$-invariance of $M_{\mathrm{F}}(\mathcal{O})$ is trivial from the definition of $\Gamma$ and the isotony property is also clear.

To prove the covariance property of the net use Lemma B.3.2 and similar arguments as in the proof of Theorem A.3.2.

For the causality choose $f=f_{1} \Theta f_{2}, h=h_{1} \oplus h_{2} \in h_{\mathrm{F}}$ such that $\operatorname{supp} f_{i} \perp \operatorname{supp} h_{i}$, $i=1,2$. Then

$$
\begin{aligned}
&\left\langle\mathfrak{I}_{\mathrm{F}}\left(f_{1} A_{1} f_{2}\right) \cdot \mathfrak{I}_{\mathrm{F}}\left(h_{1} \oplus h_{2}\right)\right\rangle_{\mathrm{F}}=\int_{\mathcal{C}^{-}}\left(\hat{f}_{1}(p), \beta_{+}(p) \hat{h}_{1}(p)\right)_{\mathcal{H}} \mu_{0}(\mathrm{~d} p)+ \\
&+\int_{c^{+}}\left(\hat{f}_{1}(-p) \cdot \beta_{-}(p) \hat{h}_{1}(-p)\right)_{\mathcal{H}} \mu_{0}(\mathrm{~d} p)+\int_{\mathcal{C}^{+}}\left(\hat{f}_{2}(-p), \beta_{-}(p) \hat{h}_{2}(-p)\right)_{\mathcal{H}} \mu_{0}(\mathrm{~d} p)+ \\
&+\int_{\mathcal{C}^{+}}\left(\hat{f}_{2}(p), \beta(p) \hat{h}_{2}(p)\right)_{\mathcal{H}} \mu_{0}(\mathrm{~d} p)=0
\end{aligned}
$$

again by $\mathrm{Eq}$. (13).

As in the massive case, using the results of Section 2, we have the following

THEOREM B.3.4. The net of $\mathrm{C}^{*}$-algebras defined by

$$
\mathbb{E}^{1} \supset \mathcal{O} \longmapsto \mathcal{A}_{F}(\mathcal{O}):=\mathrm{C}^{*}\left(\left\{A(f): \quad f \in M_{F}(\mathcal{O})\right)\right\}^{\ddot{z}_{2}}
$$

satisfies the properties (i) $)_{\mathrm{F}},(\mathrm{ii})_{\mathrm{F}}$ and $(\mathrm{iii})_{\mathrm{F}}$. 
REMARK B.3.5: Notice that a "smaller" causal and covariant net of local $\mathrm{C}^{*}$-algebras can be constructed from a certain local linear submanifold of $M_{F}(\mathcal{O})$ :

$$
\begin{aligned}
\mathbb{R}^{4} \supset \mathcal{O} & \longmapsto M_{\mathrm{F}_{1}}(\mathcal{O}) \\
& :=\operatorname{span}\left\{\mathfrak{J}_{\mathrm{F}}\left(f \in I_{0}^{\prime} f\right): f \in \mathrm{C}_{0}^{\infty}\left(\mathbb{R}^{4}, \mathcal{H}\right), \operatorname{supp} f \subset \mathcal{O}\right\} .
\end{aligned}
$$

Note that $\Gamma$ acts on elements of $M_{\mathrm{F}_{1}}(\mathcal{O})$ as the identity (i.e. we have that $\Gamma\left(\mathfrak{I}_{\mathrm{F}}\left(f \oplus \Gamma_{0} f\right)\right)=\mathfrak{I}_{\mathrm{F}}\left(f \oplus \Gamma_{0} f\right)$ for all $\left.f \in \mathrm{C}_{0}^{x}\left(\mathbb{R}^{4}, \mathcal{H}\right)\right)$, so the corresponding local algebra $\mathcal{A}_{\mathrm{F}_{1}}(\mathcal{O})$ will be generated by self-adjoint elements.

\section{B.3.3. The Bose case ( $n$ even)}

\section{B.3.3.1. CCR-algebra}

Define $h_{\mathrm{B}}:=\mathfrak{H}_{+, n}^{\prime} \oplus \mathfrak{H}_{+, n}^{\prime}$ and interpret it as a real space. Consider the following real-bilinear form: for $\varphi=\left[\varphi_{1}\right]_{+} \oplus\left[\varphi_{2}\right]_{+}, \dot{\psi}=\left[\psi_{1}\right]_{+} \oplus\left[\psi_{2}\right]_{+} \in h_{\mathrm{B}}$ let

$$
\langle\varphi, \psi\rangle_{\mathrm{B}}:=\left\langle\left[\varphi_{1}\right]_{+},\left[\psi_{1}\right]_{+}\right\rangle_{\beta_{+}}^{\prime}-\left\langle\left[\varphi_{2}\right]_{+},\left[\psi_{2}\right]_{+}\right\rangle_{\beta_{+}}^{\prime},
$$

and define a non-degenerate symplectic form

$$
\sigma(\cdot, \cdot):=\operatorname{Im}(\cdot, \cdot\rangle_{\mathrm{B}}
$$

LEMMA B.3.6. The representation $V_{\mathrm{B}}$ on $h_{\mathrm{B}}$ defined by $V_{\mathrm{B}}:=V_{1}^{\prime} \oplus V_{2}^{\prime}$ leaves the symplectic form $\sigma$ invariant.

Proof: For $[\varphi]=\left[\varphi_{1}\right]_{+} \oplus\left[\varphi_{2}\right]_{+},[\psi]=\left[\psi_{1}\right]_{+} \oplus\left[\psi_{2}\right]_{+} \in h_{\mathrm{B}}$ we have

$$
\begin{aligned}
\left\langle V_{\mathrm{B}}(g)[\varphi], V_{\mathrm{B}}(g)[\psi]\right\rangle_{\mathrm{B}}^{\prime}= & \left\langle V_{1}^{\prime}(g)\left[\psi_{1}\right]_{+}, V_{1}^{\prime}(g)\left[\psi_{1}\right]_{+}\right\rangle_{\beta_{+}}^{\prime} \\
& -\left\langle V_{2}^{\prime}(g)\left[\psi_{2}\right]_{+}, V_{2}^{\prime}(g)\left[\varphi_{2}\right]_{+}\right\rangle_{\beta_{+}}^{\prime}=\langle[\varphi],[\psi]\rangle_{\mathrm{B}}^{\prime}
\end{aligned}
$$

Therefore, $V_{\mathrm{B}}$ leaves also the symplectic form $\sigma$ invariant.

\section{B.3.3.2. Existence theorem for the local algebras}

Let $T_{1}$ and $\Im_{ \pm}$be defined as in Sections B.3.1 and B.3.2. Consider an embedding $I_{\mathrm{B}}: \mathrm{C}_{0}^{\infty}\left(\mathbb{R}^{4}, \mathcal{h}\right) \rightarrow h_{\mathrm{B}}$ defined by the equation

$$
\mathfrak{I}_{\mathrm{B}} f:=\left[\mathfrak{I}_{+} f\right]_{+} \oplus\left[\mathfrak{I}_{-} f\right]_{+}
$$

for all $f \in \mathrm{C}_{0}^{\infty}\left(\mathbb{R}^{4}, \mathcal{H}\right)$.

The covariance will be based on the following

LEMMA B.3.7. The embedding $\mathfrak{I}_{\mathrm{B}}$ intertwines between $T_{1}$ and $V_{\mathrm{B}}$ (i.e. $\mathfrak{I}_{\mathrm{B}} T_{\mathrm{J}}$ $\left.=V_{\mathrm{B}} \mathfrak{I}_{\mathrm{B}}\right)$. 
Proof: Use relations (83).

Define the net of linear submanifolds of $h_{\mathrm{B}}$ by

$$
\mathbb{R}^{4} \supset \mathcal{O} \longmapsto M_{\mathrm{B}}(\mathcal{O}):=\operatorname{span}\left\{\mathfrak{I}_{\mathrm{B}} f: \quad f \in \mathrm{C}_{0}^{\mathrm{x}}\left(\mathbb{R}^{4}, \mathcal{H}\right), \operatorname{supp} f \subset \mathcal{O}\right\} .
$$

THEOREM B.3.8. The net $\mathcal{O} \mapsto M_{\mathrm{B}}(\mathcal{O})$ satisfies the properties (I) $)_{\mathrm{B}},(\mathrm{II})_{\mathrm{B}}$ and $(\mathrm{III})_{\mathrm{B}}$ of Section 2.

Proof: Isotony is trivially satisfied and for the covariance use Lemma B.3.7 and analogous arguments as in Theorem A.3.2.

To prove the causality property, choose the test functions $f, h \in \mathrm{C}_{0}^{\infty}\left(\mathbb{R}^{4}, \mathcal{H}\right)$ such that $\operatorname{supp} f \perp \operatorname{supp} h$. Then

$$
\begin{aligned}
\left\langle\Im_{\mathrm{B}} f, \Im_{\mathrm{B}} h\right\rangle_{B}^{\prime}= & \int_{\mathcal{C}^{+}}\left(\hat{f}(p), \beta_{+}(p) \hat{h}(p)\right)_{\mathcal{H}} \mu_{0}(\mathrm{~d} p)- \\
& -\int_{\mathcal{C}^{+}}\left(\hat{f}(-p), \beta_{+}(p) \hat{h}(-p)\right)_{\mathcal{H}} \mu_{0}(\mathrm{~d} p)=0,
\end{aligned}
$$

again by Eq. (12). Therefore, $\sigma\left(\mathfrak{I}_{\mathrm{B}} f, \mathfrak{I}_{\mathrm{B}} h\right)=0$ and the net is causal.

Again, following Section 2 one has

THEOREM B.3.9. The net of $\mathrm{C}^{*}$-algebras defined by

$$
\mathbb{R}^{4} \supset \mathcal{O} \longmapsto \mathcal{A}_{\mathrm{B}}(\mathcal{O}):=\mathrm{C}^{*}\left(\left\{W(\varphi): \quad \varphi \in M_{\mathrm{B}}(\mathcal{O})\right\}\right)
$$

satisfies the properties $(\mathrm{i})_{\mathrm{B}}$, (ii) $)_{\mathrm{B}}$ and $(\text { iii })_{\mathrm{B}}$.

\section{B.4. Fock states and spectrality condition}

As in Part $A$ the Fock states for the fermionic models will be characterized by basis projections. In the Bose case we will specify an internal complexification, which characterizes the Fock state with spectrality condition.

\section{B.4.1. The Fermi case}

The statemant of the problem is analogous as in Section A.4.1. Define the following operator on $h_{\mathrm{F}}$ :

$$
P\left(\left[\varphi_{1}\right]_{+} \oplus\left[\varphi_{2}\right]_{+} \oplus\left[\varphi_{3}\right]_{-} \oplus\left[\varphi_{4}\right]_{-}\right):=\left[\varphi_{1}\right]_{+} \oplus 0 \oplus \mathbf{0} \oplus\left[\varphi_{4}\right]_{-}
$$

for all $\left[\varphi_{1}\right]_{+} \oplus\left[\varphi_{2}\right]_{+} \oplus\left[\varphi_{3}\right]_{-} \oplus\left[\varphi_{4}\right]_{-} \in h_{\mathrm{F}}$.

LEMMA B.4.1. $P$ is a basis projection, i.e. $P+\Gamma P \Gamma=\mathbb{1}$, that commutes with $V_{\mathrm{F}}$.

Proof: This is a direct consequence of the definitions of $P, \Gamma$ and $V_{\mathrm{F}}$. 
The Hilbert space $\mathfrak{H}_{P}$, associated to the representation $\pi_{P}$ characterized by the basis projection $P$, is the antisymmetric part of the Fock space constructed from $P h_{\mathrm{F}}$ $\cong \mathfrak{H}_{n,+}^{\prime} \odot \mathfrak{H}_{n,-}^{\prime} \cdot$

THEOREM B.4.2. The automorphisms $\alpha_{g}, g \in \widehat{\mathfrak{P}_{+}^{1}}$, are unitarily implementable on $\mathfrak{H}_{P}$, i.e. there exists a strongly continuous unitary representation $Q_{(\cdot)}$ of $\overline{\mathfrak{P}_{+}^{+}}$on $\mathfrak{H}_{P}$ such that

$$
\pi_{P}\left(\alpha_{g}(X)\right)=Q_{g} \pi_{P}(X) Q_{g}^{-1} \quad \text { for all } \quad X \in \operatorname{CAR}\left(h_{\mathrm{F}}, \Gamma\right) .
$$

Furthermore, for all $a \in \mathbb{R}^{4}, Q_{a}$ satisfies the spectrality condition.

Proof: The proof uses the same arguments as in the proof of Theorem A.4.2. The unitarities implementing the Bogoliubov automorphisms are now the second quantization of $V_{1}^{\prime}(g) \oplus V_{4}(g)$ on the antisymmetric part of the Fock space over $\mathfrak{H}_{P}$.

\section{B.4.2. The Bose case}

One may choose the internal complexification $J$ defined by $J\left(\left[\varphi_{1}\right]_{+} \oplus\left[\varphi_{2}\right]_{+}\right):=$ $\left[i \varphi_{1}\right]_{+} \oplus\left[-i \varphi_{2}\right]_{1}$, for all $\left[\varphi_{1}\right]_{+} \oplus\left[\varphi_{2}\right]_{+} \in h_{\mathrm{B}}$. Similar arguments as in Section A.4.2 show that this choice specifies a Fock state where the spectrality condition is satisfied. The Bogoliubov automorphisms $\alpha_{g}$ generated by $V_{\mathrm{B}}(g)$ are implemented by the second quantization of $V_{\mathrm{B}}(g)$ on the symmetric Fock space over $h_{\mathrm{B}}$, considered as a Hilbert space with the scalar product characterized by $J$.

\section{Acknowledgements}

We are indebted to Prof. H. J. Borchers for valuable remarks regarding a preliminary version of this paper. One of the authors (M. J.) would like to thank Prof. K. Fredenhagen for some useful advices concerning specification of the basis projection associated to the fermionic models.

\section{REFERENCES}

[1] R. Haag and D. Kastler: J. Math. Phys. 5 (1964), 848.

[2] R. Haag: Local Quantum Physics, Springer, Berlin 1992.

[3] G. F. dell'Antonio: Commun. Math. Phys. 9 (1968), 81.

[4] H. Araki: in: Operator Algebras and Mathematical Physics, Vol. 62 of Contemp. Math. (AMS) (1987), 23.

[5] H. Araki and M. Shiraishi: Publ. RIMS Kyoto Univ. 7 (1971/72), 105.

[6] H. Araki: Publ. RIMS Kyoto Univ. 7 (1971/72), 121.

[7] H. Baumgärtel and M. Wollenberg: Causal Nets of Operator Algebras, Mathematical Aspects of Algebraic Quantum Field Theory, Akademie Verlag, Berlin 1992.

[8] O. Bratteli and D. W. Robinson: Operator Algebras and Quantum Statistical Mechanics, Vol. II, Springer, Berlin 1981.

[9] E. P. Wigner: Ann. Math. 40 (1939), 149

[10] A. O. Barut and R. Rączka: Theory of Group Representations and Applications. Polish Scientific Publishers, Warsaw 1980. 
[11] H. Baumgärtel and M. Jurke: Preprint No. 60, SFB 288, TU Berlin, 1993.

[12] H. Baumgärtel, M. Jurke and F. Lledó: Preprint No. 120, SFB 288, TU Berlin, 1994.

[13] M. Jurke and F. Lledó: Preprint No. 137, SFB 288, TU Berlin, 1994.

[14] M. A. Neumark: Normierte Algebren. DVW, Berlin 1990.

[15] M. Reed and B. Simon: Methods of Modern Mathernatical Physics, Vol. II. AP, San Diego 1975.

[16] H. J. Borchers: in: Operator Algebras and Mathematical Physics, Vol. 62 of Contemp. Math. (AMS) (1987), 143.

[17] A. Guichardet: Symmetric Hilbert Spaces and Related Topics, Springer, Berlin 1972.

[18] B. Thaller: The Dirac Equation, Springer, Berlin 1992.

[19] D. J. Simms: Lie Groups and Quantum Mechanics, Springer, Berlin 1968.

[20] N. N. Bogoliubov, A. A. Logunov and I. T. Todorov: Introduction to Axiomatic Quantum Field Theorn, W. A. Benjamin Inc., Reading (Massachusetts) 1975.

[21] P. D. Hislop: Commun. Math. Phys. 185 (1988), 193.

122) A. O. Barut and R. Raczka: Ann. Inst. Henri Poincaré 2 (1972), 111. 